\title{
Joint integrated track splitting for multi-path multi-target tracking using OTHR detections
}

\author{
Yuan Huang ${ }^{1 \dagger}$, Taek Lyul Song ${ }^{1 * \dagger}$ (1) and Joo Hyun Lee ${ }^{2}$
}

\begin{abstract}
In target tracking applications where an over-the-horizon radar (OTHR) is used to gather measurements, one target may generate more than one measurement at each scan due to the multi-path propagation effect. However, traditional tracking methods obtain the data association probabilities based on track-to-measurement data association events under the assumption that each target can generate at most one detection at each scan, leading to poor performances if these methods are applied to multiple detection applications such as OTHR applications. In this paper, we develop a multi-path multi-target tracking algorithm entitled multiple detection joint integrated track splitting (MD-JITS). This novel algorithm jointly solves the measurement origin uncertainty and measurement path model uncertainty. The probability of target existence (PTE) is utilized in the OTHR application as a track quality measure for true track confirmation and false track discrimination. The data association algorithm of MD-JITS the proposed algorithm is realized based on measurement cells that each measurement cell consists of one or more of the validated measurements, while considering the measurement path model. The proposed algorithm is compared with the multiple detection joint integrated probabilistic data association (MD-JIPDA) algorithm in a multi-target crossing scenario, implementing the OTHR system in the presence of clutter and failed target detections, to demonstrate the desired effectiveness.
\end{abstract}

Keywords: Multi-target tracking, OTHR, Multiple detection, Track management

\section{Introduction}

The core objective for the remote sensing target localization and tracking is to generate target state estimates using measurements processed by the sensors, such as the radar [1-3]. The measurements can originate from targets or from clutter and lead to both true tracks (following the targets) and false tracks (following the clutter) existing in the surveillance area. Therefore, false track discrimination [4], involving true track confirmation and false track termination, is crucial for tracking algorithms. The probability of target existence (PTE) was first introduced in [4] as a soft decision of the existence of a target, which can be used to alleviate the false track discrimination issue.

\footnotetext{
*Correspondence: tsong@hanyang.ac.kr

†Yuan Huang and Taek Lyul Song contributed equally to this work.

${ }^{1}$ Department of Electronic Systems Engineering, Hanyang University, Hanyangdaehak-ro, Ansan, Republic of Korea

Full list of author information is available at the end of the article
}

Most target tracking algorithms assume that the sensor can generate at most one detection per target with a detection probability (usually less than unity) at each scan [5-10]. After the measurement selection procedure, multi-target tracking (MTT) algorithms will generate the joint track-to-measurement assignments across the tracks. The main difference among the MTT algorithms lies in the way assignments are realized and how the corresponding probabilities are evaluated [11].

Among various MTT algorithms, the multiple hypothesis tracking (MHT) is a theoretically optimal tracking algorithm that enumerates and evaluates all possible track-to-measurement association hypotheses [5, 12]. Each hypothesis represents a global association considering all tracks and measurements over a number of scans. Based on the different hypothesis generation criteria, different versions of MHT can be divided into two main groups. Measurement-oriented MHT [5] generates hypotheses based on measurements, while track-oriented 
MHT [12] generates hypotheses based on tracks. The maximum likelihood probabilistic multiple hypothesis tracking (ML-PMHT) is a powerful non-Bayesian algorithm that uses a generalized likelihood ratio test to confirm target existence [13]. Ciuonzo and Horn [14] use a Merkle tree to store and organize hypotheses for a distributed track-oriented multiple hypothesis tracking (TO-MHT) resulting in an efficient structure for implementation.

The joint integrated probabilistic data association (JIPDA) tracker is a single-scan MTT algorithm that incorporates the probability of target existence into the JPDA algorithm as a track quality measure [15]. JIPDA is widely used mainly because it is easy to implement and has low computational cost, due to how all the data association events for a track are compressed into one track state at each scan. In the JIPDA framework, the probability of target existence is used to solve the false track discrimination problem, and the track state is generated under the assumption of target existence. Compared to the multi-scan global hypothesis generated by the MHT tracker, the JIPDA forms track-to-measurement data association events based only on current scan circumstances, which significantly lowers the computational expense. As suggested in [12], under adverse circumstances, especially when the clutter density is high and the detection probability is low, JIPDA may have poor tracking performances. In $[16,17]$, the maximum likelihood probabilistic data association (ML-PDA) algorithm is utilized to identify the sensor states as well as target states.

The joint integrated track splitting (JITS) algorithm is a multi-scan MTT tracker [11]. Each track generates a hypotheses tree consisting of a set of hypotheses. Each hypothesis is treated as a track component, representing one assertion of the possible target detections history for multiple scans. Every component selects measurements and spawns new components, and these new components propagate to the next scan. The track state is updated by a Gaussian mixture of all the components belonging to that track. The probability of target existence is an inherent part of the tracking state and is recursively updated based on the measurement likelihood ratio, considering all the components. Updating the estimated state and the probability of target existence are two core aspects of JITS. As the number of hypotheses exponentially grows, component reduction methods are used. Pruning and merging are the efficient and most widely used methods $[18,19]$ for controlling the number of components. Both JIPDA and JITS use the probability of target existence to solve the false track discrimination issue, and the comparison results showed that JITS outperforms JIPDA at the expense of computational complexity [11]. Instead of the global hypotheses being generated by the MHT, JITS forms a hypotheses tree for each track, which significantly reduces the structure complexity. Some of the differences and tracking performance comparisons of IPDA, ITS, and MHT are shown in [20]. Kim and Song [21] proposed the smoothing JITS for multi-target tracking in clutter.

These algorithms assume that for each scan, one target can give rise to at most one detection with a given detection probability of $P_{D}<1$. However, many practical applications are plagued by the multiple detection problem. This problem is especially prominent when a special kind of radar, called the over-the-horizon radar (OTHR) [22-24], is used. In [22-24], the multi-path probabilistic data association (MPDA) method is introduced for single-target tracking. However, the multi-path tracks are generated as it lacks a means of discrimination. In the OTHR tracking system [25-28] which is widely used in remote sensing applications, transmission and receiving signals can be scattered by different ionospheric layers which results in different measurement paths (models). The multi-path approach leads to multiple detections generated by the same target, and the relationship between measurements and paths is not prior known which results in the measurement path model uncertainty. If the targets in the surveillance region are closely spaced and cross each other, localization and tracking become more difficult. Moreover, due to the incorrect model and measurement combination, multi-path tracks are generated and need to be identified. In $[25,27]$, the multiple detection multiple hypothesis tracking (MD-MHT) algorithm and the multi-detection probability hypothesis density (MDPHD) algorithm are derived by combining the multiple hypothesis tracking and the random finite set framework. In [29], the Gaussian mixture probability hypothesis density (GM-PHD) filter is extended for multi-path multitarget tracking with the over-the-horizon radar system. But the MHT and the PHD algorithms lack a specialized track quality measure for true and false track management. In $[26,28]$, the Bernoulli filter and the cardinality balanced multi-target multi-Bernoulli filter are applied to the OTHR applications. However, the Bernoulli filterbased methods are recommended to use in the environment with high detection probability and low clutter density. In [30], the authors developed the multi-radar multi-target tracking algorithm for maritime surveillance at over-the-horizon $(\mathrm{OTH})$ distances.

In multiple detection scenarios, the classic tracking algorithms, which assume at most one target originated measurement, lose a large amount of the target motion information contained in the measurements. This weakness is caused by the fact that each of the data association events only considers at most a single measurement per track. Each of the data association events assumes that one of the validated measurements is generated by a target or clutter, and then, the track state is updated based on those hypotheses. If multiple measurements can be considered 
in one date association event, the tracking performance can be improved significantly due to the target information being more efficiently extracted. The measurement partition method is introduced to generate the possible target-oriented measurement cells and was embedded in the JPDA and PHD frameworks [27, 31]. The measurement partition method assumes that more than one measurement can be generated by one target, forming all the possible target-oriented measurement combinations based both on the validated measurements and on the restriction of the maximum number of target detections. Those measurement cells are then used in the data association events instead of a single measurement. The algorithms in [27, 31] are multi-target tracking methods but do not have the track quality measure which is essential for distinguishing true and false tracks.

Both JIPDA and JITS assume that at most one measurement per target at each scan, which leads to information loss in multiple detection scenarios. By jointly considering the measurement origin uncertainty and the measurement path model uncertainty, two novel structures named multiple detection JIPDA (MD-JIPDA) and multiple detection JITS (MD-JITS) are designed especially for use in the OTHR tracking system. In these two multiple detection structures, after the measurement selection step, the measurement cells are first generated such that one measurement cell may contain one or more of these validated measurements. Then, the data association events of MD-JIPDA and MD-JITS are generated using the measurement cells. Combined with prior information from the last scan, the measurement cells are used to update the track state and the probability of target existence.

The track state of MD-JIPDA is generated by a Gaussian mixture based on all the data association events, and then, this track propagates to the next scan. The output of MD-JIPDA for each track at each scan contains one Gaussian distribution for the track state and the probability of target existence. As for MD-JITS, at each scan, each component selects measurements and generates the measurement cells separately. Each component generates new components using any one of its own measurement cells, where each component is represented by a Gaussian distribution for its state and a component probability. The final output for the track state is obtained by a Gaussian mixture based on all the components that belong to the track. Then, the components of the track propagate to the next scan where each component can be seen as a measurement cell assignment history. Compared to MD-JIPDA, in which each track propagates as one Gaussian pdf, each MD-JITS track propagates as a set of many Gaussian components. The number of components of a track exponentially increases with the number of scans, but actually not all the components are significant for the track. Thus, the components with the component probabilities lower than a given threshold are pruned, and if any two components consecutively use the same measurement cells to update the component states for several scans, these two components should be merged into one $[18,19]$. Since the MD-JITS track maintains many track components which can be seen as the target-generated measurement hypotheses, it outperforms MD-JIPDA in the multi-target crossing scenarios.

Initial results of MD-JITS are reported in [32] in which the multiple detection problem occurs from a high-resolution sensor. The algorithm proposed in [32] only needs to solve the many-to-one measurements-totrack associations. However, in order to associate multiple measurements to one track, the work proposed here should jointly consider the measurement origin uncertainty and the measurement path model uncertainty caused by the unknown signal propagation paths of the OTHR. The same measurement with different measurement path models provides completely different target state information. We provide here the rigorous algorithm derivations of MD-JITS for the OTHR system by utilizing the combinations of the measurement cells and the path models. We also introduce a multipath track discrimination method for the data association structures.

The problem statement is given in Section 2. The mathematical derivation of MD-JITS for the OTHR system and the complexity analyses are given in Section 3. Section 4 contains the simulation studies, followed by conclusions in Section 5.

In this paper, the following abbreviations, notations, and the assumption are employed:

\section{A. Abbreviations:}

CFT: Confirmed false tracks; CTTs: Confirmed true tracks; EKF: Extended Kalman filter; JIPDA: Joint integrated probabilistic data association; JITS: Joint integrated track splitting; MD-JIPDA: Multiple detection joint integrated probabilistic data association; MD-JITS: Multiple detection joint integrated track splitting; MD-MHT: Multiple detection multiple hypothesis tracking; MDPHD: Multi-detection probability hypothesis density; MLPDA: Maximum likelihood probabilistic data association; ML-PMHT: Maximum likelihood probabilistic multiple hypothesis tracking; MPDA: Multi-path probabilistic data association; MHT: Multiple hypothesis tracking; MTT: Multi-target tracking; OTHR: Over-the-horizon radar; PTE: Probability of target existence; RMSEs: Root mean square errors

\section{B. Notations:}

$\chi_{k}^{t} \quad$ The event that track $t$ is tracking a target i.e., target $t$ exists.

$m_{k}$ The number of validated measurements at $\operatorname{scan} k$. 
$\Phi_{t, \text { max }}$ The maximum number of target originated measurements in the $m_{k}$ validated measurements, $\Phi_{t, \max }=\min \left(L, m_{k}\right)$.

$z_{\Phi_{t}, n_{\Phi_{t}}}(k)$ One measurement cell of track $t$ at time $k$.

$\Phi_{t} \quad$ The number of measurements originated from target $t$ such that $1 \leq \Phi_{t} \leq \Phi_{t \text {,max }}$.

$n_{\Phi_{t}}$ An index that indicates is the measurement cell $z_{\Phi_{t}, n_{\Phi_{t}}}(k)$ is one of the possible $c_{\Phi_{t}}$ combinations where there are $\Phi_{t}$ measurements originated from target $t . n_{\Phi_{t}} \in\left\{1,2, \ldots, c_{\Phi_{t}}\right\}$ and $c_{\Phi_{t}}=C_{\Phi_{t}}^{m_{k}}=$ $\frac{m_{k} !}{\Phi_{t} !\left(m_{k}-\Phi_{t}\right) !}$ (the notation $C_{m}^{n}$ in this paper represents the number of $m$-combinations from a given set of $n$ elements).

$z(k)$ The measurement set that of all the validated measurements at scan $k, z(k)=$ $\left\{\mathbf{z}_{1}(k), \mathbf{z}_{2}(k), \ldots, \mathbf{z}_{m_{k}}(k)\right\}$.

$Z^{k} \quad$ A collection of validated measurement sets that includes all the validated measurements up to and including scan $k, Z^{k}=z(k) \cup Z^{k-1}$.

The superscript or subscript $t$ indicates that the parameter is specified by track $t$. $k$ always represents the time index.

\section{Assumption:}

There are $L$ measurement paths, and each target can be detected at most one time through each of those paths. This suggests that there are at most $L$ target measurements from each target at each scan.

\section{Problem statement}

The target motion model is given by

$$
\mathbf{x}(k)=f(k, \mathbf{x}(k-1), \mathbf{v}(k-1)) .
$$

Here, $\mathbf{x}(k)$ represents the target state at scan $k, f$ is the state propagation function, and $\mathbf{v}(k-1)$ is the process noise.

In the OTHR system, the high frequency wave reflects through ionospheric layers to detect a target located beyond the horizon. The signal is emitted by the transmitter and reflected by one of the ionosphere layers before it reaches the target, and then, it is reflected by the target and bounces off one of the ionosphere layers before it is received by the receiver. Therefore, the $m$ different ionosphere layers form $\mathrm{m}^{2}$ different measurement paths (models). In order to demonstrate the measurement geometry and measurement generation process more concisely, only two ionosphere layers are considered in this paper. Here, two assumptions are made: one is the flat earth model and the other is that the heights of the ionospheric layers are known and fixed. Those two assumptions are employed in most OTHR studies [22, 27, 31] for simplicity. In order to consider the shape of the earth, the earth is introduced as an ellipsoid based on the WGS-84 coordinate system.
Then, the spherical OTHR measurement geometry can be modeled according to this coordinate system [33]. In realistic situations, the ionosphere state evolves intermittently. The noisy height information can be introduced by tacking into account that the ionosphere state changes slowly and stays invariable within the sampling period. Then, OTHR target tracking with the noisy ionosphere state is performed by joint estimation of fast-updating multi-target states and intermittent-updating the ionosphere state [34-36].

Figure 1 demonstrates the geometry of the planar OTHR measurement model. The receiver is set as the origin, and the transmitter is installed along the $X$-axis with a distance $d$ (T-R distance) away from the origin. The targets move in the $X-Y$ plane, and the $X-Y-Z$ coordinate system follows the right-hand rule. The ground range between the target and the receiver is $\rho$, and the bearing between the $Y$-axis and $\rho$ is defined as $b . \theta$ is the angle between the $X$-axis and the received signal satisfies $\cos \theta=\cos \varphi \sin b$, where the signal elevation $\varphi$ is not measured but has the relation $\cos \varphi=\rho /\left(2 r_{1}\right)$ with the target state $\rho$ if the target state $\mathbf{x}(k)$ in (1) consists of the ground range, ground range rate, bearing, and bearing rate $\mathbf{x}(k)=[\rho, \dot{\rho}, b, \dot{b}]^{T}$. The apparent azimuth is defined by $\pi / 2-\theta$. The transmit and receive signals are reflected by the layers with heights $h_{t}$ and $h_{r}$, respectively. $h_{t}$ and $h_{r}$ are used to represent the transmit layer and the receive layer, respectively. Half of the slant ranges from the transmitter to target, and from the target to receiver are denoted by $r_{2}$ and $r_{1}$, respectively.

Here, two ionospheric layers are considered with vertical heights $h_{E}$ and $h_{F} . h_{E}$ and $h_{F}$ are used to represent the layer $E$ and layer $F$, respectively. Under this circumstance, the signal propagation models are shown in the Table 1 in which the height of the ionospheric layer is used to represent the layer.

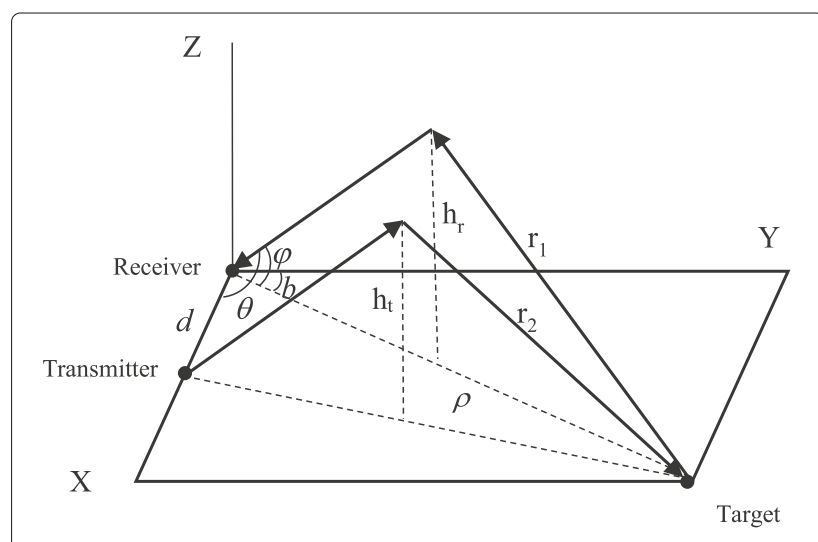

Fig. 1 Geometry of the planar OTHR measurement model 
Table 1 Propagation models

\begin{tabular}{llll}
\hline Path & Model & Transmit layer $h_{t}$ & Receive layer $h_{r}$ \\
\hline$i=1$ & $E E$ & $h_{E}$ & $h_{E}$ \\
$i=2$ & $E F$ & $h_{E}$ & $h_{F}$ \\
$i=3$ & $F E$ & $h_{F}$ & $h_{E}$ \\
$i=4$ & $F F$ & $h_{F}$ & $h_{F}$ \\
\hline
\end{tabular}

The measurements generated by the target and clutter are given by

$$
\mathbf{z}_{i}(k)=\left\{\begin{array}{cc}
h_{E E}(\mathbf{x}(k))+\mathbf{w}_{E E}(k) \text { model EE, Path } \\
h_{E F}(\mathbf{x}(k))+\mathbf{w}_{E F}(k) \text { model EF, Path } \\
\\
h_{F E}(\mathbf{x}(k))+\mathbf{w}_{F E}(k) \text { model FE, Path } \\
h_{F F}(\mathbf{x}(k))+\mathbf{w}_{F F}(k) & \text { model FF, Path } \\
c & \text { clutter }
\end{array}\right.
$$

where $h_{t r}(t, r \in\{E, F\})$ is the measurement function associated with the transmit layer $t$ and receive layer $r . \mathbf{w}_{t r}$ $(t, r \in\{E, F\})$ is the zero mean Gaussian measurement noise in the corresponding paths. Both $h_{t r}$ and $\mathbf{w}_{t r}$ are related to the corresponding model.

The elements of each measurement consist of the slant range, the rate of change of the slant range, and the apparent azimuth $\left[R_{g}, R_{r}, A_{z}\right]$ [22], given by

$$
\begin{aligned}
& R_{g}=r_{1}+r_{2} \\
& R_{r}=\frac{\dot{\rho}}{4}\left\{\frac{\rho}{r_{1}}+\frac{\eta}{r_{2}}\right\}, \\
& A_{z}=\sin ^{-1}\left\{\rho \sin (b) /\left(2 r_{1}\right)\right\}
\end{aligned}
$$

where the parameters $r_{1}, r_{2}$, and $\eta$ are obtained by

$$
\begin{aligned}
r_{1} & =r_{1}\left(\rho, h_{r}\right) \triangleq \sqrt{(\rho / 2)}^{2}+h_{r}^{2} \\
r_{2} & =r_{2}\left(\rho, b, h_{r}\right) \triangleq \sqrt{(\rho / 2)^{2}-d \rho \sin (b) / 2+(d / 2)^{2}+h_{t}^{2}} \\
\eta & =\rho-d \sin (b),
\end{aligned}
$$

where $h_{t}$ and $h_{r}$ represent the height of transmit layer and receive layer associated with different measurement models.

\section{Multiple detection JITS}

In the MD-JITS data association step, one has to consider the possibility that multiple measurements might originate from the same target. This special issue of the MD-JITS data association process involves jointly assigning the measurement cells to the tracks. At each scan, each component of each track employs the gating method $[1,2]$ to validate measurements. After obtaining validated measurements, the measurement partition method is used to generate the measurement cells from these validated measurements. Each measurement cell contains one or some of the validated measurements. Then, a measurement cell assigned to a tracker should not share any elements with other tracks in a feasible joint event [3].

The joint events are used to jointly assign the measurement cells to the tracks, and each joint event is composed of tracks with disjoint measurement cells. In a joint event, each track is assigned to one measurement cell or unassigned. Then, the joint event probabilities are calculated for the corresponding joint events.

\subsection{Measurement partition method}

In order to assign multiple measurements to one track, the measurement cells are generated. Each measurement cell can be treated as a measurement set which contains at most $\Phi_{t \text {,max }}$ elements of the validated measurements. In a data association event, if a measurement cell is assigned to a track, the measurements contained in this measurement cell are used for the state estimation and the data association probability calculation. The measurement cells are generated using the validated measurements based on the assumption that there are $\Phi_{t}$ measurements originated from target $t$.

Here, we use an example to show the process of generating the measurement cells. Suppose that track $t$ validates three measurements $\left\{\mathbf{z}_{1}(k), \mathbf{z}_{2}(k), \mathbf{z}_{4}(k)\right\}$ out of the total cluster measurements $\left\{\mathbf{z}_{1}(k), \mathbf{z}_{2}(k), \mathbf{z}_{3}(k), \mathbf{z}_{4}(k)\right\}$ at scan $k, \Phi_{t, \max }$ is set to be 3 . Then, based on the assumed number of measurements from the target, measurement cells are generated as follows:

- Suppose that only one of the validated measurements is the target detection $\left(\Phi_{t}=1\right)$ and $n_{1} \in\{1,2,3\}$. The measurement cells are:

$$
\begin{aligned}
& z_{1,1}(k)=\left\{\mathbf{z}_{1}(k)\right\} ; \\
& z_{1,2}(k)=\left\{\mathbf{z}_{2}(k)\right\} ; \\
& z_{1,3}(k)=\left\{\mathbf{z}_{4}(k)\right\} .
\end{aligned}
$$

- Suppose that two of the validated measurements are the target detections $\left(\Phi_{t}=2\right)$ and $n_{2} \in\{1,2,3\}$. The measurement cells are:

$$
\begin{aligned}
& z_{2,1}(k)=\left\{\mathbf{z}_{1}(k), \mathbf{z}_{2}(k)\right\} ; \\
& z_{2,2}(k)=\left\{\mathbf{z}_{1}(k), \mathbf{z}_{4}(k)\right\} ; \\
& z_{2,3}(k)=\left\{\mathbf{z}_{2}(k), \mathbf{z}_{4}(k)\right\} .
\end{aligned}
$$

- Suppose that all these three measurements are the target detections $\left(\Phi_{t}=3\right)$ and $n_{3} \in\{1\}$. The measurement cell is:

$$
z_{3,1}(k)=\left\{\mathbf{z}_{1}(k), \mathbf{z}_{2}(k), \mathbf{z}_{4}(k)\right\} .
$$

After the measurement partition process, seven measurement cells are generated. These measurement cells are 
utilized in the data associations for track $t$. When measurement cell $z_{2,2}(k)$ is considered in a data association event, the measurements contained in $z_{2,2}(k)$, which are $\mathbf{z}_{1}(k)$ and $\mathbf{z}_{4}(k)$, are treated as target detections.

The number of measurement cells increases with the number of validated measurements $m_{k}$ and the maximum number of target originated measurements $\Phi_{t, \max }$. After the measurement partition process, the number of measurement cells becomes $\sum_{\Phi_{t}=1}^{\Phi_{t, \max }} C_{\Phi_{t}}^{m_{k}}$.

Here, the measurement cells are generated without considering the measurement cell path model. The measurement cell path model, which contains path models for each measurement in the measurement cell, will be specified in Section 3.2.

\subsection{MD-JITS}

When there are tracks that share measurements, the feasible joint events are used to solve the track-tomeasurement assignment issue. The feasible joint events cover all possible track-to-measurement assignments for all the tracks and the measurements in a cluster. A cluster at scan $k$ is a set of the tracks and the measurements these tracks select. A track inside the cluster should share measurements with one or more different tracks in the cluster, and the tracks not belonging to the cluster do not select any of the cluster measurements. In the multiple detection case, the measurement cells composed of different validated measurements are assigned to the tracks in one feasible joint event.

In this paper, for reasons of clarity and without loss of generality, it is assumed that all the measurements in the cluster are validated by all the tracks belonging to that cluster and that the detection probability of each path is considered identical, i.e., $P_{D E E}=P_{D E F}=P_{D F E}=P_{D F F}=P_{D}$.

MD-JITS is different from MD-JIPDA in the sense that each track in MD-JITS retains the track components for propagation. Denote by

$$
\begin{gathered}
\kappa^{t}(k)=\left\langle z_{\Phi_{t}, n_{\Phi_{t}}}(k) \mid M_{j_{k}}^{t}\left(z_{\Phi_{t}, n_{\Phi_{t}}}(k)\right)\right\rangle, \\
j_{k} \in\left\{1,2, \ldots, \frac{L !}{\left(L-\Phi_{t}\right) !}\right\}
\end{gathered}
$$

for the event that measurement cell $z_{\Phi_{t}, n_{\Phi_{t}}}(k)$ is composed of the $\Phi_{t}$ measurements of track $t$ generated by a path model $M_{j_{k}}^{t}\left(z_{\Phi_{t}, n_{\Phi_{t}}}(k)\right) . M_{j_{k}}^{t}\left(z_{\Phi_{t}, n_{\Phi_{t}}}(k)\right)$ is the hypothesis that $z_{\Phi_{t}, n_{\Phi_{t}}}(k)$ is originated from one of $\frac{L !}{\left(L-\Phi_{t}\right) !}$ possible model path allocations. For example, under the condition that $L=4$ for the measurement cell $z_{2,2}(k)=\left\{\mathbf{z}_{1}(k), \mathbf{z}_{4}(k)\right\}$, the corresponding measurement cell model $M_{j_{k}}^{t}\left(z_{2,2}(k)\right)$ can be chosen from

$$
\begin{array}{r}
\{(1,2),(2,1),(1,3),(3,1),(1,4),(4,1),(2,3),(3,2), \\
(2,4),(4,2),(3,4),(4,3)\} .
\end{array}
$$

If the measurement cell model is chosen as $(3,4)$, this means that measurement $\mathbf{z}_{1}(k)$ is generated by path 3 and measurement $\mathbf{z}_{4}(k)$ is generated by path 4 in Table 1 . In OTHR target tracking applications, the measurements are used with the specified path models since the same measurement with different path models provides different target state information.

Denote by

$$
\kappa^{t}(k)=\langle 0 \mid 0\rangle
$$

the event that none of the validated measurements are the detections of target $t$.

One possible history of the validated measurement cells for track $t$ during the interval between scan 1 and scan $k$ is represented by a set of events that represents a sequence of the measurement cells and the corresponding path model in the interval:

$$
\begin{aligned}
\kappa^{t, k} \triangleq \quad\left\{\kappa^{t}(1)\right. & =\left\langle z_{\Phi_{t}, n_{\Phi_{t}}}(1) \mid M_{j_{1}}^{t}\left(z_{\Phi_{t}, n_{\Phi_{t}}}(1)\right)\right\rangle, \\
\ldots, \kappa^{t}(k) & \left.=\left\langle z_{\Phi_{t}, n_{\Phi_{t}}}(k) \mid M_{j_{k}}^{t}\left(z_{\Phi_{t}, n_{\Phi_{t}}}(k)\right)\right\rangle\right\},
\end{aligned}
$$

where each $\kappa^{t}(i), i \in\{1,2, \ldots, k\}$ can be of the form from (5) or (6). Each $\kappa^{t, k}$ represents one component for track $t$ at time $k$.

Here, one recursion of MD-JITS at scan $k$ is illustrated. At scan $k$, the following posterior information at scan $k-1$ is assumed to be available: (1) The track state probability density function (pdf) for track t, $p\left(\mathbf{x}^{t}(k-1) \mid \chi_{k-1}^{t}, Z^{k-1}\right)$; (2) the probability of target existence for track $t, P\left(\chi_{k-1}^{t} \mid Z^{k-1}\right)$, which represents the probability of target existence of a target tracked by track $t$; (3) the component state for each component of track $t$, $p\left(\mathbf{x}^{t}(k-1) \mid \chi_{k-1}^{t}, \kappa^{t, k-1}, Z^{k-1}\right)$; and (4) the component probability for each component belongs to track $t$, which is $P\left(\kappa^{t, k-1} \mid \chi_{k-1}, Z^{k-1}\right)$.

The prediction and the measurement selection processes are implemented by each component of track $t$. Here, track $t$ represents one of the existing or the initiated tracks at scan $k$. The component state prediction is governed by

$$
\begin{aligned}
& p\left(\mathbf{x}^{t}(k) \mid \chi_{k}^{t}, \kappa^{t, k-1}, Z^{k-1}\right) \\
& =\int_{\mathbf{x}^{t}(k-1)} p\left(\mathbf{x}^{t}(k) \mid \mathbf{x}^{t}(k-1), \chi_{k}^{t}, \kappa^{t, k-1}, Z^{k-1}\right) 4 \\
& \quad \cdot p\left(\mathbf{x}^{t}(k-1) \mid \chi_{k}^{t}, \kappa^{t, k-1}, Z^{k-1}\right) d \mathbf{x}^{t}(k-1),
\end{aligned}
$$


where the Kalman filter prediction can be used for a linear state propagation model. If $\kappa^{t, k-1}$ is omitted, this equation can be used for MD-JIPDA track state prediction.

The component probability prediction is given as

$$
P\left(\kappa^{t, k-1} \mid \chi_{k}^{t}, Z^{k-1}\right)=P\left(\kappa^{t, k-1} \mid \chi_{k-1}^{t}, Z^{k-1}\right)
$$

the details can be found in $[12,20]$.

The following describes the measurement selection of each component of track $t$. Each component of track $t$ selects the measurements in its validation gate, and all the components of track $t$ form a common validation gate and common validated measurements of track $t$ $[20,37]$. Based on (8) and the selection process of the validated measurements, each component of track $t$ generates the predicted measurement for each measurement Path and selects the measurements inside the validation gate corresponding to $\mathrm{Path}_{i}$ using an ellipse gating method [3].

After selecting the measurements, the measurement partition for the set of validated measurements is applied to generate the measurement cells for possible multiple detection. Then, the component measurement cell likelihood function for each measurement cell is calculated as

$$
\begin{aligned}
& p\left(z_{\Phi_{t}, n_{\Phi_{t}}}(k) \mid \chi_{k}^{t}, \kappa^{t, k-1}, M_{j_{k}}^{t}\left(z_{\Phi_{t}, n_{\Phi_{t}}}(k)\right), Z^{k-1}\right) \\
= & \frac{N\left(z_{\Phi_{t}, n_{\Phi_{t}}}(k) \mid \zeta_{M_{j_{k}}^{t}\left(z_{\Phi_{t}, n_{\Phi_{t}}}(k)\right)}, \mathbf{S}_{M_{j_{k}}^{t}}\left(z_{\Phi_{t}, n_{\Phi_{t}}}(k)\right)\right)}{\left(P_{G}\right)^{\Phi_{t}}} .
\end{aligned}
$$

In (10), $P_{G}$ is the gating probability of a single detection. The Gaussian pdf for measurement cell $z_{\Phi_{t}, n_{\Phi_{t}}}(k)$ is employed, and each measurement cell is allocated with one measurement cell path model $M_{j_{k}}^{t}\left(z_{\Phi_{t}, n_{\Phi_{t}}}(k)\right)$. Note that $\zeta_{M_{j_{k}}^{t}\left(z_{\Phi_{t}, n_{\Phi_{t}}}(k)\right)}$ represents the measurement prediction based on the state prediction (8) and measurement cell path model $M_{j_{k}}^{t}\left(z_{\Phi_{t}, n_{\Phi_{t}}}(k)\right)$. Note also that $\mathbf{S}_{M_{j_{k}}^{t}\left(z_{\Phi_{t}, n_{\Phi_{t}}}(k)\right)}$ represents the measurement cell innovation covariance corresponding to measurement cell $z_{\Phi_{t}, n_{\Phi_{t}}}(k)$ given measurement cell path model $M_{j_{k}}^{t}\left(z_{\Phi_{t}, n_{\Phi_{t}}}(k)\right)$. One can apply the extended Kalman filter (EKF) to obtain state estimates for the equations. The details for generating these estimates can be found in $[22,23]$.

Equation (10) is the measurement cell likelihood function calculated for each component. The combined measurement cell likelihood of track $t$ for measurement cell $z_{\Phi_{t}, n_{\Phi_{t}}}(k)$ with allocated path model $M_{j_{k}}^{t}\left(z_{\Phi_{t}, n_{\Phi_{t}}}(k)\right)$ is the sum of products of the component measurement cell likelihood function and the component probability of the track components of track $t$, written as

$$
\begin{aligned}
& p\left(z_{\Phi_{t}, n_{\Phi_{t}}}(k) \mid \chi_{k}^{t}, M_{j_{k}}^{t}\left(z_{\Phi_{t}, n_{\Phi_{t}}}(k)\right), Z^{k-1}\right) \\
& =\sum_{\kappa^{t, k-1}} p\left(z_{\Phi_{t}, n_{\Phi_{t}}}(k) \mid \chi_{k}^{t}, \kappa^{t, k-1}, M_{j_{k}}^{t}\left(z_{\Phi_{t}, n_{\Phi_{t}}}(k)\right), Z^{k-1}\right) \\
& \quad P\left(\kappa^{t, k-1} \mid \chi_{k}^{t}, Z^{k-1}\right),
\end{aligned}
$$

where this combined measurement likelihood function utilizes all the components that use measurement cell $z_{\Phi_{t}, n_{\Phi_{t}}}(k)$ with the allocated path model $M_{j_{k}}^{t}\left(z_{\Phi_{t}, n_{\Phi_{t}}}(k)\right)$. This combined measurement cell likelihood is used to calculate the joint event probability.

Then, the data association step is processed. In the following derivations, "track" and "measurement" mean cluster tracks and cluster measurements in a cluster, respectively. The validated measurement set $z(k)$ is the union of the validated measurements validated by each of the tracks belonging to the cluster. A joint event $\varepsilon$ is an event of assigning measurement cells including nondetection to all the tracks. One joint event should satisfy the following: (1) Each track is assigned to at most one measurement cell and (2) each measurement cell is assigned to at most one track.

To generate the a posteriori probability of the joint event $\varepsilon$, the tracks are divided into different sets. $T^{\varepsilon}$ is the set of tracks that are assigned a measurement cell, and the number of tracks in this set is $N^{\varepsilon}$. $T_{0}^{\varepsilon}$ is the set of tracks that are not assigned measurement cells (i.e., assigned to nondetection), and the number of tracks in this set is $N_{0}^{\varepsilon}$. The a posteriori probability of the joint event $\varepsilon$, using Bayes' formula, is

$$
\begin{aligned}
P\left(\varepsilon \mid Z^{k}\right) & =\frac{p\left(\varepsilon, z(k), m_{k} \mid Z^{k-1}\right)}{p\left(z(k) \mid Z^{k-1}\right)} \\
& =\frac{1}{c} p\left(z(k) \mid \varepsilon, m_{k}, Z^{k-1}\right) P\left(m_{k} \mid \varepsilon, Z^{k-1}\right) P\left(\varepsilon \mid Z^{k-1}\right),
\end{aligned}
$$

where $c$ is a normalization constant and $m_{k}$ is the number of validated measurements in the cluster. $p\left(z(k) \mid \varepsilon, m_{k}, Z^{k-1}\right)$ is the joint measurement likelihood function for measurement set $z(k) ; P\left(m_{k} \mid \varepsilon, Z^{k-1}\right)$ represents the a priori probability for the number of the measurements; and $P\left(\varepsilon \mid Z^{k-1}\right)$ is the a priori probability of the joint event.

The joint measurement likelihood $p\left(z(k) \mid \varepsilon, m_{k}, Z^{k-1}\right)$ of $z(k)$ in (12) is calculated by

$$
\begin{aligned}
& p\left(z(k) \mid \varepsilon, m_{k}, Z^{k-1}\right)=V_{k}^{-\left(m_{k}-\sum_{t=1}^{N^{\varepsilon}} \Phi_{t}\right)} \\
& \cdot \prod_{t \in T^{\varepsilon}} p\left(z_{\Phi_{t}, n_{\Phi_{t}}}(k) \mid \chi_{k}^{t}, M_{j_{k}}^{t}\left(z_{\Phi_{t}, n_{\Phi_{t}}}(k)\right), Z^{k-1}\right),
\end{aligned}
$$

where $V_{k}$ is the volume of the surveillance region. 
For the joint event $\varepsilon$, the relation between measurement cell $z_{\Phi_{t}, n_{\Phi_{t}}}(k)$ and measurement cell path model $M_{j_{k}}^{t}\left(z_{\Phi_{t}, n_{\Phi_{t}}}(k)\right)$ for every track involved in $\varepsilon$ is already known.

The number of clutter measurements follows a Poisson distribution [38]. Given $m_{k}$ measurements at scan $k$, $m_{k}-\sum_{t=1}^{N^{\varepsilon}} \Phi_{t}$ is the number of clutter-generated measurements. The probability that $m_{k}-\sum_{t=1}^{N^{\varepsilon}} \Phi_{t}$ clutter measurements are generated at scan $k$ is given by

$$
P\left(m_{k}-\sum_{t=1}^{N^{\varepsilon}} \Phi_{t} \mid Z^{k-1}\right)=e^{-\hat{m}_{k}} \frac{\left(\hat{m}_{k}\right)^{m_{k}-\sum_{t=1}^{N^{\varepsilon}} \Phi_{t}}}{\left(m_{k}-\sum_{t=1}^{N^{\varepsilon}} \Phi_{t}\right) !}
$$

where $\hat{m}_{k}$ is the mean number of clutter measurements such that $\hat{m}_{k}=\lambda V_{k}$ with the clutter measurement density $\lambda$.

In (12), the a priori probability of the joint event $\varepsilon$ is given by

$$
\begin{aligned}
& P\left(\varepsilon \mid Z^{k-1}\right) \\
& =c_{1}{ }^{-1} P\left(\bigcap_{t \in T_{0}^{\varepsilon}} \varepsilon_{k, 0}^{t} \bigcap_{t \in T^{\varepsilon}}\left(\chi_{k}^{t}, \varepsilon_{\left.k,\left|z_{\Phi_{t}, n_{\Phi_{t}}}^{t}(k)\right| M_{j_{k}}^{t}\left(z_{\Phi_{t}, n_{\Phi_{t}}}(k)\right)\right)}\right) \mid Z^{k-1}\right) \\
& =c_{1}{ }^{-1} \prod_{t \in T_{0}^{\varepsilon}}\left[1-P_{D e c}^{t} P\left(\chi_{k}^{t} \mid Z^{k-1}\right)\right] \\
& \cdot P\left(\bigcap_{t \in T^{\varepsilon}} \varepsilon_{k,\left|z_{\Phi_{t}, n_{\Phi_{t}}}^{t}(k)\right| M_{j_{k}}^{t}\left(z_{\Phi_{t, n}, \Phi_{t}}(k)\right) \mid} \mid \bigcap_{t \in T^{\varepsilon}} \chi_{k}^{t}, Z^{k-1}\right) \\
& \cdot P\left(\bigcap_{t \in T^{\varepsilon}} \chi_{k}^{t} \mid Z^{k-1}\right) \text {. }
\end{aligned}
$$

In (15), the a priori probability of the event that no validated measurement is generated by target $t$ including the hypothesis of non existence of target $t$ at scan $k$ is

$$
P\left(\varepsilon_{k, 0}^{t} \mid Z^{k-1}\right)=1-P_{D e c}^{t} P\left(\chi_{k}^{t} \mid Z^{k-1}\right),
$$

where $P\left(\chi_{k}^{t} \mid Z^{k-1}\right)$ is the predicted probability of the existence of target $t$. The probability that there is at least one target detection in the validation gate is given by $P_{D e c}=$ $1-\left(1-P_{D} P_{G}\right)^{L}$.

In (15), $P\left(\chi_{k}^{t} \mid Z^{k-1}\right)$ is the predicted probability of target existence. The propagation of the probability of target existence for each target is modeled as a Markov chain $[4,15]$. The existence probability of target $t$ and the nonexistence probability at time $k$ are denoted by $P\left(\chi_{k}^{t} \mid Z^{k}\right)$ and $P\left(\bar{\chi}_{k}^{t} \mid Z^{k}\right)$, respectively. The predicted existence probabilities are denoted as $P\left(\chi_{k}^{t} \mid Z^{k-1}\right)$ and $P\left(\bar{\chi}_{k}^{t} \mid Z^{k-1}\right)$, respectively. The Markov chain model defines two events $\chi_{k}^{t}$ and $\bar{\chi}_{k}^{t}$ by a random variable $e_{k}^{t}$ such that $e_{k}^{t}=1$ represents $\chi_{k}^{t}$ and $e_{k}^{t}=2$ represents $\bar{\chi}_{k}^{t}$. The predicted state of target existence from scan $k-1$ to scan $k$ is given by

$$
\left[\begin{array}{l}
P\left(\chi_{k}^{t} \mid Z^{k-1}\right) \\
P\left(\bar{\chi}_{k}^{t} \mid Z^{k-1}\right)
\end{array}\right]=\left[\begin{array}{ll}
P_{11} & P_{21} \\
P_{12} & P_{22}
\end{array}\right]\left[\begin{array}{c}
P\left(\chi_{k-1}^{t} \mid Z^{k-1}\right) \\
P\left(\bar{\chi}_{k-1}^{t} \mid Z^{k-1}\right)
\end{array}\right] .
$$

Equation (17) represents the prediction process for the probability of target existence where $P_{i j}=P\left[e_{k}^{t}=j \mid e_{k-1}^{t}=i\right], j \in\{1,2\}$ represents the transitional probability between the existence states. $P_{i j}$ satisfies $\sum_{j=1}^{2} P_{i j}=1$ for $i \in\{1,2\}$.

In (15), the a priori probability that the assignment is correct becomes

$$
\begin{aligned}
& P\left(\bigcap_{t \in T^{\varepsilon}} \varepsilon_{k,\left|z_{\Phi_{t}, n_{\Phi_{t}}}^{t}(k)\right| M_{j_{k}}^{t}\left(z_{\Phi_{t}, n_{\Phi_{t}}}(k)\right) \mid} \mid \bigcap_{t \in T^{\varepsilon}} \chi_{k}^{t}, Z^{k-1}\right) \\
& =\frac{1}{C_{\Phi_{1}}^{m_{k}} C_{\Phi_{2}}^{m_{k}-\Phi_{1}} \ldots C_{\Phi_{N^{\varepsilon}}}^{m_{k}-\sum_{i=1}^{N^{\varepsilon}-1} \Phi_{i}}} \prod_{t \in T^{\varepsilon}} \frac{\left(L-\Phi_{t}\right) !}{L !} \prod_{t \in T^{\varepsilon}} P_{D G \Phi_{t}} \\
& =\frac{\left(m_{k}-\sum_{i=1}^{N^{\varepsilon}} \Phi_{i}\right) !}{m_{k} !} \prod_{t \in T^{\varepsilon}} \bar{P}_{D G \Phi_{t}} \text {, }
\end{aligned}
$$

where $P_{D G \Phi_{t}}$ is the probability that there are $\Phi_{t}$ measurements detected and fall into the gate, given by

$$
\begin{aligned}
P_{D G \Phi_{t}} & =\frac{L !}{\Phi_{t} !\left(L-\Phi_{t}\right) !}\left(P_{D} P_{G}\right)^{\Phi_{t}}\left(1-P_{D} P_{G}\right)^{L-\Phi_{t}} \\
& \triangleq \frac{L !}{\Phi_{t} !\left(L-\Phi_{t}\right) !} \bar{P}_{D G \Phi_{t}} .
\end{aligned}
$$

Combining (13), (14), and (15) yields the a posteriori probability of the joint association event $\varepsilon$ as

$$
\begin{aligned}
& P\left(\varepsilon \mid Z^{k}\right) \\
& =c^{-1} V_{k}^{-\left(m_{k}-\sum_{t=1}^{N^{\varepsilon}} \Phi_{t}\right)} e^{-\hat{m}_{k}} \frac{\left(m_{k}\right)^{m_{k}-\sum_{t=1}^{N^{\varepsilon}} \Phi_{t}}}{\left(m_{k}-\sum_{t=1}^{N^{\varepsilon}} \Phi_{t}\right) !} \frac{\left(m_{k}-\sum_{t=1}^{N^{\varepsilon}} \Phi_{t}\right) !}{m_{k} !} \\
& \cdot \prod_{t \in T_{0}^{\varepsilon}}\left[1-P_{D e c}^{t} P\left(\chi_{k}^{t} \mid Z^{k-1}\right)\right] \\
& \cdot \prod_{t \in T^{\varepsilon}}\left[p\left(z_{\Phi_{t}, n_{\Phi_{t}}}(k) \mid M_{j_{k}}^{t}\left(z_{\Phi_{t}, n_{\Phi_{t}}}(k)\right), Z^{k-1}\right) \bar{P}_{D G \Phi_{t}} P\left(\chi_{k}^{t} \mid Z^{k-1}\right)\right] \\
& =C^{-1} \prod_{t \in T_{0}^{\varepsilon}}\left[1-P_{D e c}^{t} P\left(\chi_{k}^{t} \mid Z^{k-1}\right)\right] \\
& \cdot \prod_{t \in T^{\varepsilon}}\left[\frac{p\left(z_{\Phi_{t}, n_{\Phi_{t}}}(k) \mid \chi_{k}^{t}, M_{j_{k}}^{t}\left(z_{\Phi_{t}, n_{\Phi_{t}}}(k)\right), Z^{k-1}\right)}{\lambda^{\Phi_{t}}} \bar{P}_{D G \Phi_{t}} P\left(\chi_{k}^{t} \mid Z^{k-1}\right)\right] .
\end{aligned}
$$


Then, those joint events and the corresponding event probabilities are used to generate the data association probabilities of measurement cell-to-track assignments for MD-JIPDA and MD-JITS.

Denote by $\Xi\left(\varepsilon,\left\langle z_{\Phi_{t}, n_{\Phi_{t}}}(k) \mid M_{j_{k}}^{t}\left(z_{\Phi_{t}, n_{\Phi_{t}}}(k)\right)\right\rangle\right)$ the set of all the joint events that allocate measurement cell $z_{\Phi_{t}, n_{\Phi_{t}}}(k)$ to track $t$ with measurement cell path model $M_{j_{k}}^{t}\left(z_{\Phi_{t}, n_{\Phi_{t}}}(k)\right)$. The probability that non-detection is assigned to track $t$ is the probability of the union of all joint events that assign non-detection to track $t$, given by

$$
P\left(\kappa^{t}(k)=\langle 0 \mid 0\rangle \mid Z^{k}\right)=\sum_{\varepsilon \in \Xi(t,\langle 0 \mid 0\rangle)} P\left(\varepsilon \mid Z^{k}\right) .
$$

The probability that no measurement in the cluster is generated by target $t$ under the assumption that target $t$ exists is given as

$$
\begin{aligned}
P\left(\chi_{k}^{t}, \kappa^{t}(k)=\right. & \left.\langle 0 \mid 0\rangle \mid Z^{k}\right) \\
= & P\left(\chi_{k}^{t} \mid \kappa^{t}(k)=\langle 0 \mid 0\rangle, Z^{k}\right) P\left(\kappa^{t}(k)=\langle 0 \mid 0\rangle \mid Z^{k}\right) \\
= & \frac{P\left(\chi_{k}^{t}, z(k) \mid \kappa^{t}(k)=\langle 0 \mid 0\rangle, Z^{k-1}\right)}{P\left(z(k) \mid \kappa^{t}(k)=\langle 0 \mid 0\rangle, Z^{k-1}\right)} \\
& \cdot P\left(\kappa^{t}(k)=\langle 0 \mid 0\rangle \mid Z^{k}\right) \\
= & \frac{\left(1-P_{D e c}^{t}\right) p\left(\chi_{k}^{t} \mid Z^{k-1}\right)}{1-P_{\text {Dec }}^{t} p\left(\chi_{k}^{t} \mid Z^{k-1}\right)} P\left(\kappa^{t}(k)=\langle 0 \mid 0\rangle \mid Z^{k}\right) .
\end{aligned}
$$

The details for deriving (22) can be found in [11, 20].

Then, the probability that measurement cell $z_{\Phi_{t}, n_{\Phi_{t}}}(k)$ with measurement cell path model $M_{j_{k}}^{t}\left(z_{\Phi_{t}, n_{\Phi_{t}}}(k)\right)$ is target $t$ detection (detection of object $t$ implies existence of object $t$ ) is

$$
\begin{aligned}
& P\left(\chi_{k}^{t}, \kappa^{t}(k)=\left\langle z_{\Phi_{t}, n_{\Phi_{t}}}(k) \mid M_{j_{k}}^{t}\left(z_{\Phi_{t}, n_{\Phi_{t}}}(k)\right)\right\rangle \mid Z^{k}\right) \\
& =\sum_{\left.\varepsilon \in \Xi\left(t,\left|z_{\Phi_{t}, n_{\Phi_{t}}}(k)\right| M_{j_{k}}^{t}\left(z_{\Phi_{t}, n_{\Phi_{t}}}(k)\right)\right\rangle\right)} P\left(\varepsilon \mid Z^{k}\right) .
\end{aligned}
$$

The events that contain $\left\{\chi_{k}^{t}, \kappa^{t}(k)\right\}$ are mutually exclusive, and their union is the event of target existence. Therefore, the a posteriori probability of target existence is

$$
P\left(\chi_{k}^{t} \mid Z^{k}\right)=\sum_{\kappa^{t}(k)} P\left(\chi_{k}^{t}, \kappa^{t}(k) \mid Z^{k}\right),
$$

where $\sum_{\kappa^{t}(k)}$ denotes the summation through all feasible joint events that contain the measurement cells with the allocated measurement cell path models of track $t$ including $\kappa^{t}(k)=\langle 0 \mid 0\rangle$. The probability of non-existence $P\left(\bar{\chi}_{k}^{t} \mid Z^{k}\right)$ is equal to $1-P\left(\chi_{k}^{t} \mid Z^{k}\right)$.
The data association probabilities are given by

$$
\begin{gathered}
\beta_{k}^{t}(\langle 0 \mid 0\rangle)=P\left(\kappa^{t}(k)=\langle 0 \mid 0\rangle \mid \chi_{k}^{t}, Z^{k}\right) \\
=\frac{P\left(\chi_{k}^{t}, \kappa^{t}(k)=\langle 0 \mid 0\rangle \mid Z^{k}\right)}{P\left(\chi_{k}^{t} \mid Z^{k}\right)}, \\
\beta_{k}^{t}\left(\left\langle z_{\Phi_{t}, n_{\Phi_{t}}}(k) \mid M_{j_{k}}^{t}\left(z_{\Phi_{t}, n_{\Phi_{t}}}(k)\right)\right\rangle\right) \\
=P\left(\kappa^{t}(k)=\left\langle z_{\Phi_{t}, n_{\Phi_{t}}}(k) \mid M_{j_{k}}^{t}\left(z_{\Phi_{t}, n_{\Phi_{t}}}(k)\right)\right\rangle \mid \chi_{k}^{t}, Z^{k}\right) \\
=\frac{P\left(\chi_{k}^{t}, \kappa^{t}(k)=\left\langle z_{\Phi_{t}, n_{\Phi_{t}}}(k) \mid M_{j_{k}}^{t}\left(z_{\Phi_{t}, n_{\Phi_{t}}}(k)\right)\right\rangle \mid Z^{k}\right)}{P\left(\chi_{k}^{t} \mid Z^{k}\right)} .
\end{gathered}
$$

So far, we have obtained the data association probabilities for track-to-measurement cell assignments for all the cluster tracks. In MD-JIPDA, each data association event generates one track a posteriori state pdf denoted by $p\left(\mathbf{x}^{t}(k) \mid \chi_{k}^{t}, \kappa^{t}(k), Z^{k}\right)$, which can be obtained from the existing nonlinear filters, such as the EKF. The state for track $t$ in MD-JIPDA is obtained by summing the probabilities of all the data association events related to track $t$ under the assumption of target existence, given as

$$
\begin{aligned}
& p\left(\mathbf{x}^{t}(k) \mid \chi_{k}^{t}, Z^{k}\right) \\
& \quad=\sum_{\kappa^{t}(k)} p\left(\mathbf{x}^{t}(k) \mid \chi_{k}^{t}, \kappa^{t}(k), Z^{k}\right) P\left(\kappa^{t}(k) \mid \chi_{k}^{t}, Z^{k}\right) .
\end{aligned}
$$

The component probability for $\left\{\kappa^{t}(k)=\langle 0 \mid 0\rangle, \kappa^{t, k-1}\right\}$ is

$$
\begin{aligned}
P\left(\kappa^{t, k} \mid \chi_{k}^{t}, Z^{k}\right) & =P\left(\kappa^{t}(k)=\langle 0 \mid 0\rangle, \kappa^{t, k-1} \mid \chi_{k}^{t}, Z^{k}\right) \\
& =\beta_{k}^{t}(\langle 0 \mid 0\rangle) P\left(\kappa^{k-1} \mid \chi_{k}^{t}, Z^{k-1}\right) .
\end{aligned}
$$

The component probability for $\left\{\kappa^{t}(k)=\left\langle z_{\Phi_{t}, n_{\Phi_{t}}}(k)\right.\right.$

$$
\begin{aligned}
&\left.\left|M_{j_{k}}^{t}\left(z_{\Phi_{t}, n_{\Phi_{t}}}(k)\right)\right\rangle, \kappa^{t, k-1}\right\} \text { is } \\
& P\left(\kappa^{t, k} \mid \chi_{k}^{t}, Z^{k}\right)= P\left(\kappa^{t, k-1} \mid \chi_{k}^{t}, \kappa^{t}(k)=\left\langle z_{\Phi_{t}, n_{\Phi_{t}}}(k) \mid M_{j_{k}}^{t}\left(z_{\Phi_{t}, n_{\Phi_{t}}}(k)\right)\right\rangle, Z^{k}\right) \\
& \cdot P\left(\kappa^{t}(k)=\left\langle z_{\Phi_{t}, n_{\Phi_{t}}}(k)\left|M_{j_{k}}^{t}\left(z_{\Phi_{t}, n_{\Phi_{t}}}(k)\right)\right|\right| \chi_{k}^{t}, Z^{k}\right) \\
&= \frac{p\left(z_{\Phi_{t}, n_{\Phi_{t}}}(k) \mid \chi_{k}^{t}, \kappa^{t, k-1}, M_{j_{k}}^{t}\left(z_{\Phi_{t}, n_{\Phi_{t}}}(k)\right), Z^{k-1}\right)}{p\left(z_{\Phi_{t}, n \Phi_{t}}(k) \mid \chi_{k}^{t}, M_{j_{k}}^{t}\left(z_{\Phi_{t}, n_{\Phi_{t}}}(k)\right), Z^{k-1}\right)} \\
& \cdot P\left(\kappa^{t, k-1} \mid \chi_{k}^{t}, Z^{k-1}\right) \beta_{k}^{t}\left(\left|z_{\Phi_{t}, n_{\Phi_{t}}}(k)\right| M_{j_{k}}^{t}\left(z_{\Phi_{t}, n_{\Phi_{t}}}(k)\right) \mid\right),
\end{aligned}
$$

where the likelihood functions $p\left(z_{\Phi_{t}, n_{\Phi_{t}}}(k) \mid \chi_{k}^{t}, \kappa^{t, k-1}\right.$, $\left.M_{j_{k}}^{t}\left(z_{\Phi_{t}, n_{\Phi_{t}}}(k)\right), Z^{k-1}\right) \quad$ and $\quad p\left(z_{\Phi_{t}, n_{\Phi_{t}}}(k) \mid \chi_{k}^{t}, M_{j_{k}}^{t}\right.$ 
$\left.\left(z_{\Phi_{t}, n_{\Phi_{t}}}(k)\right), Z^{k-1}\right)$ are presented in (10) and (11), respectively.

The track state is updated using a Gaussian mixture of all the component state pdfs, given by

$$
p\left(\mathbf{x}^{t}(k) \mid \chi_{k}^{t}, Z^{k}\right)=\sum_{\kappa^{t, k}} p\left(\mathbf{x}^{t}(k) \mid \chi_{k}^{t}, \kappa^{t, k}, Z^{k}\right) P\left(\kappa^{t, k} \mid \chi_{k}^{t}, Z^{k}\right),
$$

where $p\left(\mathbf{x}^{t}(k) \mid \chi_{k}^{t}, \kappa^{t, k}, Z^{k}\right)$ is the a posteriori state pdf for each component, which can be updated by the existing nonlinear filters. If the EKF is used, the details to calculate the Jacobian matrix can be found in [22, 23]. $P\left(\kappa^{t, k} \mid \chi_{k}^{t}, Z^{k}\right)$ is the a posteriori component probability, presented in either (28) or (29) depending on context. Equation (30) is different from Eq. (27) since the association history, which is $\kappa^{t, k}$, is considered in (30) and the association at current scan, which is $\kappa^{t}(k)$, is considered in (27).

For comparison, the pseudo-codes for MD-JITS and MD-JIPDA are given in Table 2.

\subsection{Complexity analyses}

Multiple detection multi-target tracking algorithms allow for many-to-one measurements-to-track assignments in the joint events. The number of feasible joint assignments (i.e., the number of the joint events) is combinatorial in the number of the measurements and the number of the tracks. In multiple detection multi-target tracking, most of the computational load is generated by these joint assignments. The computational load generated by this part is identical in MD-JITS and MD-JIPDA, because the same joint assignment process is used in these two algorithms.

The main difference between MD-JITS and MD-JIPDA lies in the track structure. In MD-JITS, each track is modeled as a set of track components that each component has a unique measurement assignment history that consists of zero or some of the validated measurements at each scan. A track is the union of its mutually exclusive components. However, each track in MD-JIPDA is expressed by one Gaussian pdf. The track structure difference makes MDJITS more robust but more time-consuming compared to MD-JIPDA.

In order to analyze the complexity of MD-JITS, the joint measurements-to-track assignments and the track structure are discussed.

\section{A. Complexity of joint measurements-to-track assign-} ments

In Section 3, we have shown the measurement cell generation process for a track $t$. Here, we analyze the complexity of joint measurements-to-track assignments considering all the validated measurements with the specified
Table 2 Pseudo-codes for MD-JITS and MD-JIPDA

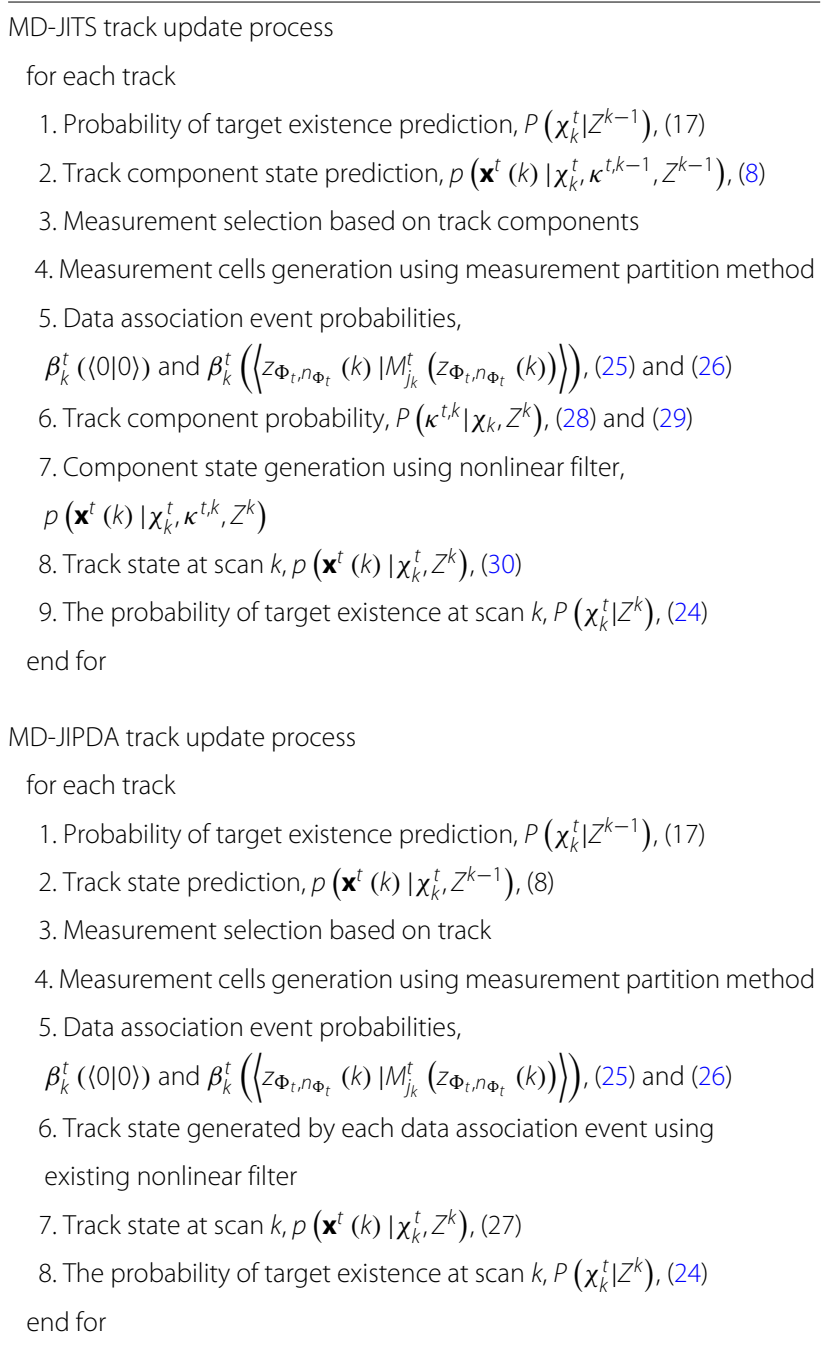

path models and all the cluster tracks. We assume that all the validated measurements are shared among the cluster tracks, and there are $M_{k}$ path-assigned measurements that satisfies

$$
M_{k} \geq \sum_{t=1}^{N} \Phi_{t, \max }
$$

where $N$ is the number of tracks considered.

Each joint event assigns zero or some of the $M_{k}$ pathassigned measurements to each of the $N$ cluster tracks. The total number of feasible joint events becomes

$$
\sum_{l_{1}=0}^{\Phi_{1, \max }} \sum_{l_{2}=0}^{\Phi_{2, \max }}, \ldots, \sum_{l_{N}=0}^{\Phi_{N, \max }}\left[C_{l_{1}}^{M_{k}} C_{l_{2}}^{M_{k}-l_{1}} \ldots C_{l_{N}}^{M_{k}-\sum_{j=1}^{N-1} l_{j}}\right]
$$

where $l_{t}(t=1, \cdots, N)$ represents the number of the pathassigned measurements allocated to track $t$. 
Suppose that $\Phi_{t, \max }=3$. In Table 3, the numbers of joint events for different $M_{k}$ and different numbers of tracks are shown. In this table, the number of joint events exponentially grows with the number of tracks and the number of the path-assigned measurements. $\Phi_{t, \max }$ is a parameter predetermined by the designer, and it also has an influence on the number of the joint events.

\section{B. Complexity of the track structure}

In this part, the complexities of the track propagation of a track in MD-JITS and MD-JIPDA are analyzed.

Let $B_{k}$ denote the number of the path-assigned measurement cells represented by $\left\langle z_{\Phi_{t}, n_{\Phi_{t}}}(k)\right| M_{j_{k}}^{t}$ $\left.\left(z_{\Phi_{t}, n_{\Phi_{t}}}(k)\right)\right\rangle$ including the special case $\langle 0 \mid 0\rangle$. In MDJITS, each measurement cell and path model combination $\left(\left\langle z_{\Phi_{t}, n_{\Phi_{t}}}(k) \mid M_{j_{k}}^{t}\left(z_{\Phi_{t}, n_{\Phi_{t}}}(k)\right)\right\rangle\right.$ or $\left.\langle 0 \mid 0\rangle\right)$ is combined to the parent component $\kappa^{t, k-1}$ to make a new component. Thus, component $\kappa^{t, k-1}$ becomes $B_{k}$ new components after processing the combinations. Finally, the total number of new components becomes $K \cdot B_{k}$ after processing all the combinations with all the $K$ parent components. For each component, as well as for each track, the state estimate and the a posteriori component probability are computed recursively. The MD-JITS track propagation has the complexity of $\mathcal{O}\left(K \cdot B_{k}\right)$ at scan $k$. The complexity of this algorithm increases as the increasing of the number of measurement.

The number of components grows exponentially in time. Practical implementation of MD-JITS must include procedures to control the number of components. Component merging and pruning techniques have been described in [37].

Each track in MD-JIPDA keeps only one Gaussian pdf, representing the track state estimate. At scan $k, B_{k}$ combinations are associated to a track. This association problem can be solved with the complexity $\mathcal{O}\left(B_{k}\right)$.

\section{Results and discussion}

In this section, the simulation performances of MDJIPDA and MD-JITS are compared. In total, 250 Monte Carlo simulation runs are employed, where each run comprises 40 scans. Four targets exist from the initial scan to

Table 3 Number of joint events

\begin{tabular}{llll}
\hline$M_{k}$ & \multicolumn{3}{l}{ Number of tracks } \\
\cline { 2 - 4 } & 1 & 2 & 3 \\
\hline 9 & 130 & 6799 & 136,348 \\
10 & 176 & 8980 & 398,476 \\
11 & 232 & 11,603 & $1,079,464$ \\
12 & 299 & 14,710 & $2,715,541$ \\
\hline
\end{tabular}

the final scan, and they propagate according to a nearly constant velocity (NCV) model. In each of the simulation runs, the influence of process noise is added to the true target trajectories, as shown in Fig. 2. Target 1 and target 2 intersect at scan 30. Both MD-JIPDA and MD-JITS use the same measurement set for the simulations.

The OTHR system geometry is as shown in Fig. 1, and the information on the surveillance area is given in Table 4. The average number of the clutter measurements at each scan is set to be 25. The NCV model for target dynamics is employed as

$$
\mathbf{x}(k)=\mathbf{F} \mathbf{x}(k-1)+\mathbf{v}(k-1),
$$

where $\mathbf{x}(k)$ is the target state at scan $k$, the sampling interval is $20 \mathrm{~s}, \mathbf{F}$ is the state propagation matrix, and $v(k-1)$ is the zero-mean Gaussian process noise with covariance Q. The process noise covariance is given by

$$
\begin{array}{r}
\mathbf{Q}=\operatorname{blockdiag}\left(\left[\begin{array}{l}
7.8 \times 10^{-1}, 4.4 \times 10^{-4} \\
4.4 \times 10^{-4}, 1.3 \times 10^{-5}
\end{array}\right],\right. \\
\left.\left[\begin{array}{l}
1.5 \times 10^{-12}, 1.1 \times 10^{-13} \\
1.1 \times 10^{-13}, 1.1 \times 10^{-14}
\end{array}\right]\right),
\end{array}
$$

The target measurement $\left[R_{g}, R_{r}, A_{z}\right]$ in $(\mathrm{km}, \mathrm{km} / \mathrm{s}, \mathrm{rad})$ is generated by (3) including the zero-mean Gaussian measurement noise with covariance $R$ given by

$$
\mathbf{R}=\operatorname{diag}\left(25 \mathrm{~km}^{2}, 1 \times 10^{-6} \mathrm{~km}^{2} / \mathrm{s}^{2}, 9 \times 10^{-6} \mathrm{rad}^{2}\right) \text {. }
$$

The target state contains information on the range, range rate, bearing, and bearing rate $\mathbf{x}(k)=\left[\begin{array}{llll}\rho & \dot{\rho} & b & b\end{array}\right]^{T}$, and the initial target states are given in Table 5 . The target detection probability of each path $P_{D}$ and the gating probability $P_{G}$ are given in Table 5 .

The initial track state covariance, which is needed in the track initialization step, is given by

$$
\begin{aligned}
\mathbf{P}_{0 \mid 0}= & \operatorname{diag}\left(25 \mathrm{~km}^{2}, 1 \times 10^{-5} \mathrm{~km}^{2} / \mathrm{s}^{2}, 9 \times 10^{-6} \mathrm{rad}^{2},\right. \\
& \left.6.4 \times 10^{-8} \mathrm{rad}^{2} / \mathrm{s}^{2}\right) .
\end{aligned}
$$

The rate of bearing is set to be 0 in the initialization step. Some of those parameters are the same as given in [39].

The transitional probabilities used in the Markov chain process are given by

$$
\left[\begin{array}{ll}
P_{11} & P_{21} \\
P_{12} & P_{22}
\end{array}\right]=\left[\begin{array}{ll}
0.98 & 0 \\
0.02 & 1
\end{array}\right]
$$

In order to initialize tracks, the inverse mapping of the measurements from radar coordinates to target motion coordinates is given by 


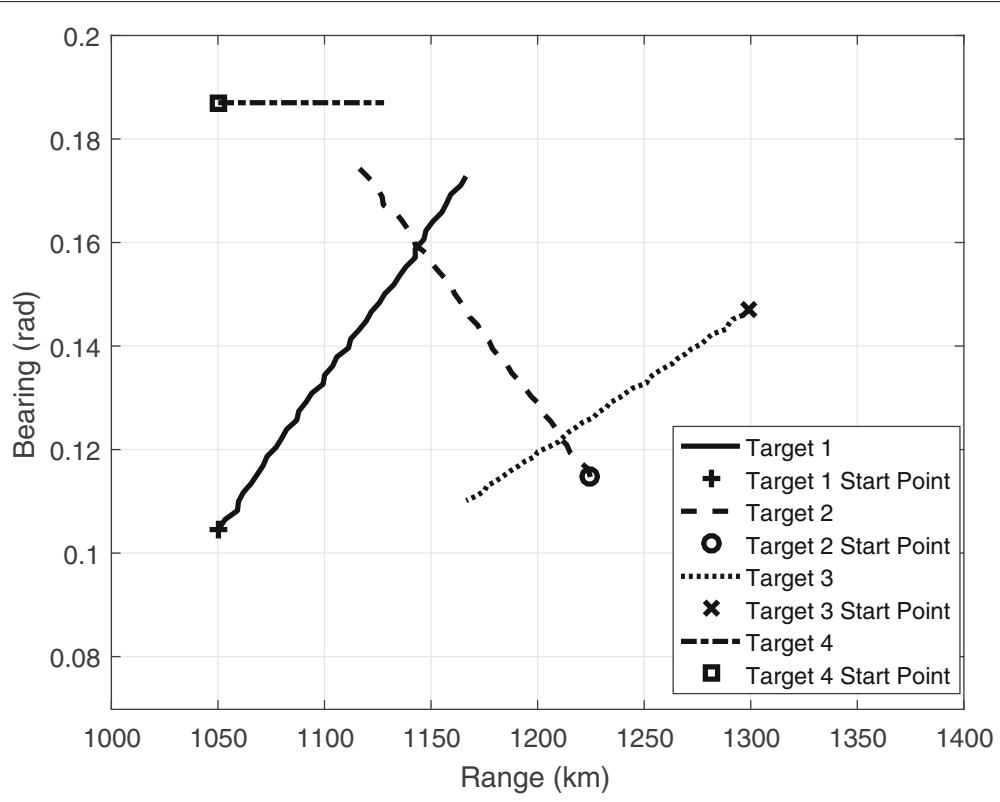

Fig. 2 True target trajectories

$$
\left\{\begin{array}{l}
\rho=2 \sqrt{r_{1}^{2}-h_{r}^{2}} \\
\dot{\rho}=4 R_{r} / r_{1}+\left(\eta / r_{2}\right)\left(\rho / r_{1}+\eta / r_{2}\right) \\
b=\sin ^{-1}\left(\frac{\sin \left(A_{z}\right)}{\cos (\varphi)}\right)
\end{array}\right.
$$

where

$$
\left\{\begin{array}{l}
r_{1}=\frac{R_{g}^{2}+h_{r}^{2}-h_{t}^{2}-(d / 2)^{2}}{2 R_{g}-d \sin \left(A_{z}\right)} \\
r_{2}=R_{g}-r_{1}
\end{array}\right.
$$

\subsection{False track discrimination and track accuracy}

The tracking performance is tested with emphasis on the false track discrimination and tracking accuracy. Both MD-JIPDA and MD-JITS use the probability of target existence to solve the false track discrimination issue as follows:

- A track is initialized by the one-point initialization method. Each track is assigned an initial probability of target existence in the track initialization step. The values of the initial probability of target existence for
MD-JIPDA tracks and MD-JITS tracks are different, ensuring that those two trackers have the same number of confirmed false tracks.

- The probability of target existence of a track is updated scan by scan, and once it exceeds the confirmation probability of target existence (0.98), the track becomes a confirmed track.

- The confirmed track is tested as to whether it is a confirmed true track or a confirmed false track by the following criteria:

$(\tilde{\mathbf{x}}(k \mid k))^{T} \mathbf{P}_{0 \mid 0}^{-1} \tilde{\mathbf{x}}(k \mid k)<20$ for comfirmed true track, $(\tilde{\mathbf{x}}(k \mid k))^{T} \mathbf{P}_{0 \mid 0}^{-1} \tilde{\mathbf{x}}(k \mid k)>40$ for comfirmed false track.

Here, $\tilde{\mathbf{x}}(k \mid k)=\mathbf{x}(k)-\hat{\mathbf{x}}(k \mid k)$ is the state estimation error of a confirmed track at time $k$ and $\mathbf{P}_{0 \mid 0}$ is the initial state covariance given in (32).

- If the probability of target existence of a track is lower than the termination probability of target existence
Table 4 Surveillance area parameters

\begin{tabular}{ll}
\hline Parameter & Value \\
\hline Slant range size & $1000-1400 \mathrm{~km}$ \\
Rate of slant range size & $0.013889-0.22222 \mathrm{~km} / \mathrm{s}$ \\
Apparent azimuth size & $0.069813-0.17453 \mathrm{rad}$ \\
T to R distance $d$ & $100 \mathrm{~km}$ \\
Hight of layer $E$ & $100 \mathrm{~km}$ \\
Hight of layer $F$ & $260 \mathrm{~km}$ \\
\hline
\end{tabular}

Table 5 Target parameters

\begin{tabular}{ll}
\hline Parameter Value \\
\hline Target1 & {$\left[1050 \mathrm{~km} ; 0.15 \mathrm{~km} / \mathrm{s} ; 0.10472 \mathrm{rad} ; 8.72665 \times 10^{-5} \mathrm{rad} / \mathrm{s}\right]$} \\
Target2 & {$\left[1225 \mathrm{~km} ;-0.14 \mathrm{~km} / \mathrm{s} ; 0.11472 \mathrm{rad} ; 7.72665 \times 10^{-5} \mathrm{rad} / \mathrm{s}\right]$} \\
Target3 & {$\left[1300 \mathrm{~km} ;-0.17 \mathrm{~km} / \mathrm{s} ; 0.14701 \mathrm{rad} ;-4.72665 \times 10^{-5} \mathrm{rad} / \mathrm{s}\right]$} \\
Target4 & {$[1050 \mathrm{~km} ; 0.1 \mathrm{~km} / \mathrm{s} ; 0.18701 \mathrm{rad} ; 0 \mathrm{rad} / \mathrm{s}]$} \\
$P_{D}$ & 0.4 \\
$P_{G}$ & 0.9966
\end{tabular}


(initial probability of target existence/5), the track is terminated.

The simulation parameters used for MD-JITS and MDJIPDA are given in Table 6. For the fair comparison, the confirmation probabilities of target existence (confirmation PTE) of MD-JIPDA and MD-JITS are set to be the same and the initial probabilities of target existence (initial PTE) of these two algorithms are adjusted so that both of these two algorithms obtain the same number of confirmed false tracks (CFT).

The simulation performances are given as in Fig. 3 through Fig. 5. Since the tracking performances for these four targets are very similar, only performances for target 2 and target 3 are shown in this paper. The numbers of the confirmed true tracks (CTTs) of both algorithms in 250 Monte Carlo runs are shown in Fig. 3 for target 2 and target 3 . The numbers of confirmed true tracks of MD-JIPDA and MD-JITS are compared under the condition that both of the two trackers have the same number of confirmed false tracks (=9). These two figures indicate that MD-JITS has a faster confirmation rate for obtaining 250 confirmed true tracks (100 percent) compared to MD-JIPDA.

Figure 4 depicts the RMSEs (root mean square errors) in the range estimation for target 2 and target 3 . In earlier scans, there is a small difference between these two trackers, while both the trackers eventually maintain the same level of estimation accuracy.

The RMSEs in the bearing of target 2 and target 3 are shown in Fig. 5. The RMSEs in the bearing first increase due to the bearing rate being set as 0 in the track initialization step, and so both of these two trackers need some time to recover this parameter. Then, the estimation error in the bearing estimation is reduced as the number of scans increases. All these simulation results demonstrate the benefits of using MD-JITS. MD-JITS outperforms MD-JIPDA, especially in terms of false track discrimination, while MD-JITS maintains the same estimation accuracy in terms of RMSE in both range and bearing estimates.

This simulation is implemented on a $4.00 \mathrm{GHz}$, Intel Core i7 PC and run with MATLAB. The CPU time per each Monte Carlo run for MD-JIPDA and MD-JITS are $345.7360 \mathrm{~s}$ and $349.3160 \mathrm{~s}$, respectively. The simulation

Table 6 Tracker parameters

\begin{tabular}{lll}
\hline & MD-JIPDA & MD-JITS \\
\hline Initial PTE & 0.000001 & 0.0017 \\
Confirmation PTE & 0.98 & 0.98 \\
Termination PTE & $0.000001 / 5$ & $0.0017 / 5$ \\
Number of CFT & 9 & 9
\end{tabular}

time is similar since most of the computational load is assigned to the joint measurements-to-track assignment part, which is identical for both MD-JIPDA and MD-JITS. Since MD-JITS needs to maintain track components, the computational cost of MD-JITS is a little bit higher than that of MD-JIPDA.

All the simulation results suggest that MD-JITS has better true track confirmation performances compared to MD-JIPDA. However, in order to achieve this benefit, MD-JITS is more time-consuming.

\subsection{A measure for the multi-path track discrimination}

In the OTHR system, there is a special kind of tracks called the multi-path track [39]. The multi-path track is generated due to an incorrect combination between the measurements and the propagation models at each scan. The multi-path track is different from the false track since the multi-path track uses target measurement to update the track state and propagate parallel to the true target trajectory, while the false track is updated by using the clutter measurement and wanders away from the true target trajectory. Thus, one of the tasks when using the OTHR tracking system is to distinguish between the multi-path track and the true track. The mechanism analysis details are given in [26]. Following this, we propose a method for both MD-JIPDA and MD-JITS to distinguish the multipath tracks.

The maximum number of measurement paths (models) for each target is defined as $L$. Here, we define a variable vector $\left[a_{k, 1}^{t}, a_{k, 2}^{t}, \ldots, a_{k, L}^{t}\right]\left(a_{k, i}^{t} \in\{0,1\}, i=1,2, \ldots L\right)$ that is used to count the active paths at scan $k$ for track $t$. $a_{k, i}^{t}=1$ means that path $i$ is active at scan $k$, and $a_{k, i}^{t}=0$ means that path $i$ is not active at scan $k$. In MD-JIPDA, the state estimation is generated by a Gaussian mixture considering all of the data association events, where each event represents the track state pdf for the track involved in the event. Among all the data association events of a track, the one with the highest data association probability is selected and the measurement path models considered in this data association event are counted. For example, in the event that has the highest data association probability, it turns out that $\mathbf{z}_{1}(k)$ and $\mathbf{z}_{3}(k)$ are considered as target detections. $\mathbf{z}_{1}(k)$ comes from path 1 and $\mathbf{z}_{3}(k)$ comes from path 2 ; thus, the corresponding active model vector is $\left[a_{k, 1}^{t}, a_{k, 2}^{t}, a_{k, 3}^{t}, a_{k, 4}^{t}\right]=[1,1,0,0]$ (path 1 and path 2 are active). As for MD-JITS, the measurement path models considered in the component with the highest component probability are counted. Then, after each simulation run, we obtain a statistic of the active paths over all the scans $\left[a_{1}^{t}, a_{2}^{t}, \ldots, a_{L}^{t}\right]$, where $a_{i}^{t}=\sum_{k} a_{k, i}^{t}$. The active path statistic $\left[a_{1}^{t}, a_{2}^{t}, \ldots, a_{L}^{t}\right]$ is used to distinguish between the true track and the multi-path track. The proposed method is used in Section 4 to discriminate the multi-path tracks. 


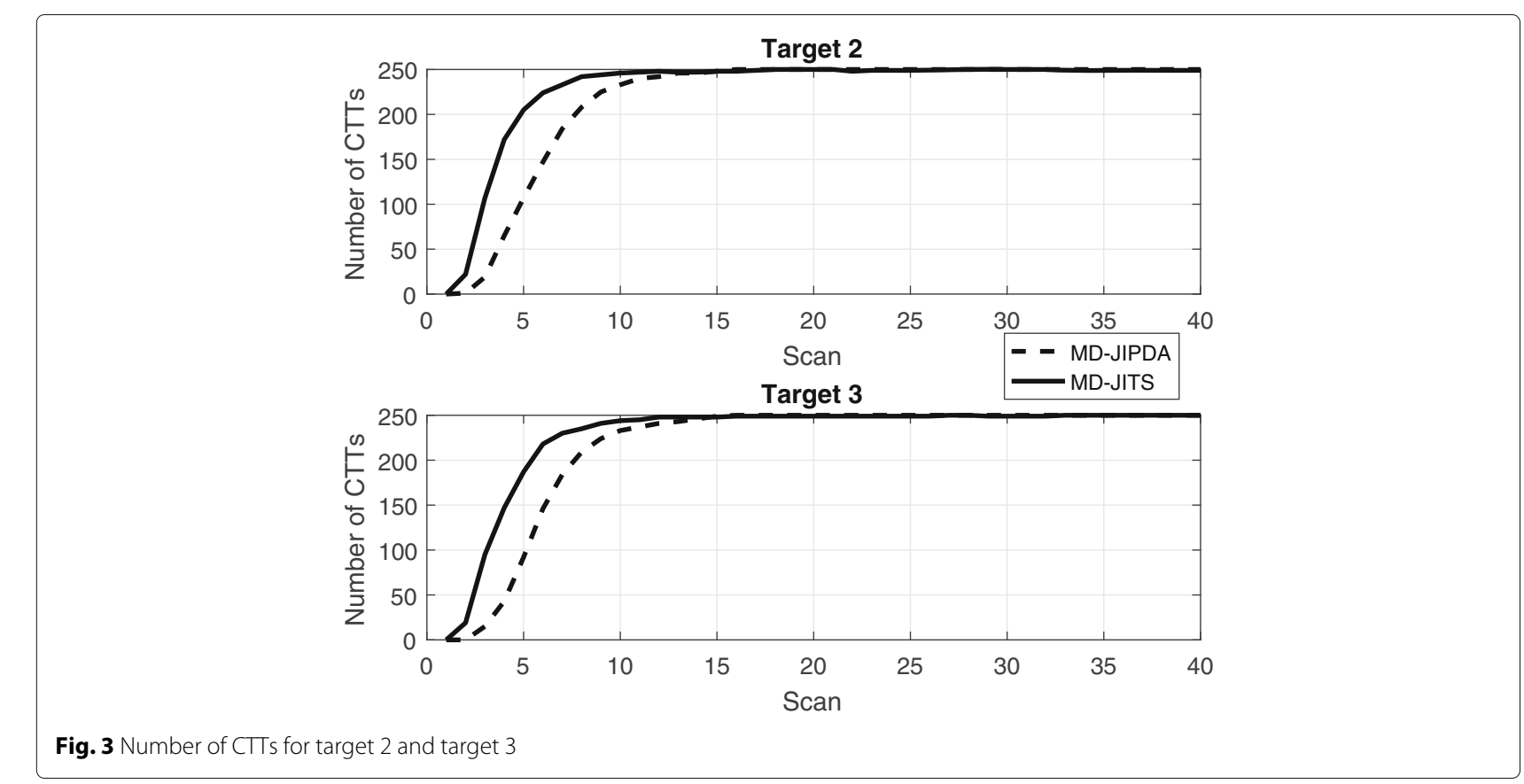

Multi-path tracks exist in this surveillance scenario, and the trajectories for two tracks (one is the true track and another is the multi-path track) for one simulation for MD-JITS are shown in Fig. 6. In this figure, the track T1 represents the true track for target 2 and T2 represents the multi-path track for target 2 . In Table 7 , the estimated active model calculated by the method introduced in this section is listed. From the table, we can see that the active mode values of the multi-path track are significantly different across the models, which can be used to distinguish the multi-path tracks from the true tracks. Given that $P_{D}=0.4$ for target measurement from each path, the accumulated path statistic of $T 1$ in Table 7 shows almost 16 detections in 40 scans for each measurement path. However, $T 2$ does not show this tendency.
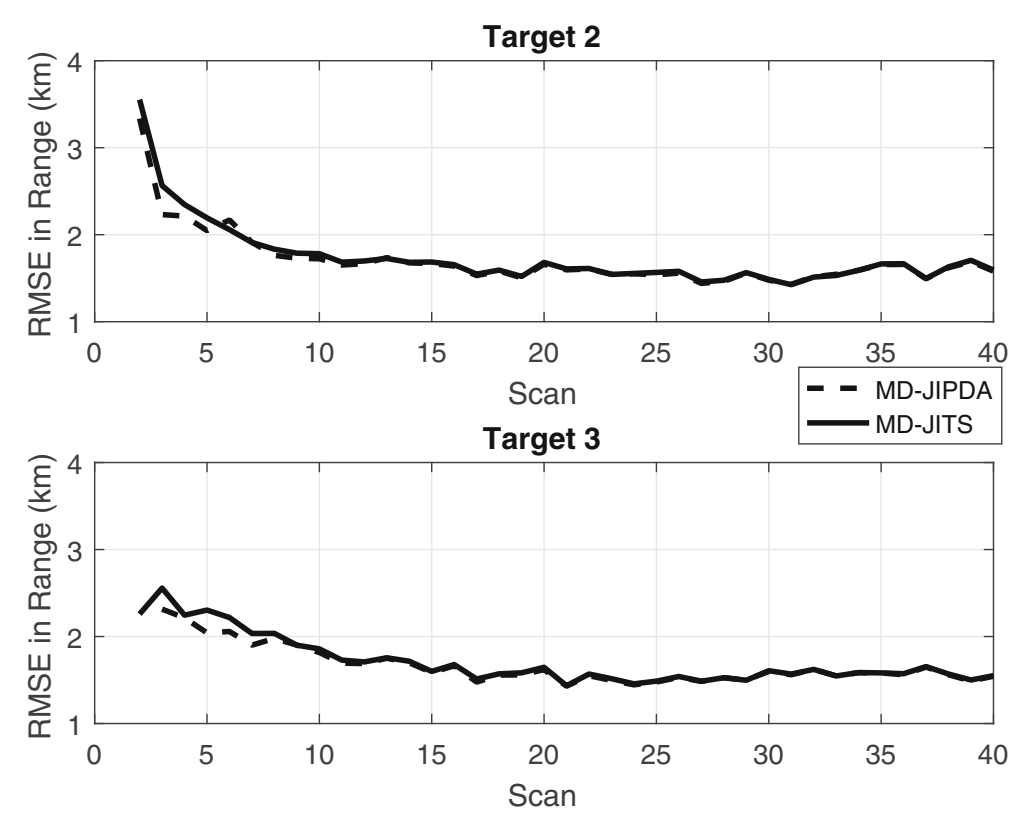

Fig. 4 RMSE in range for target 2 and target 3 


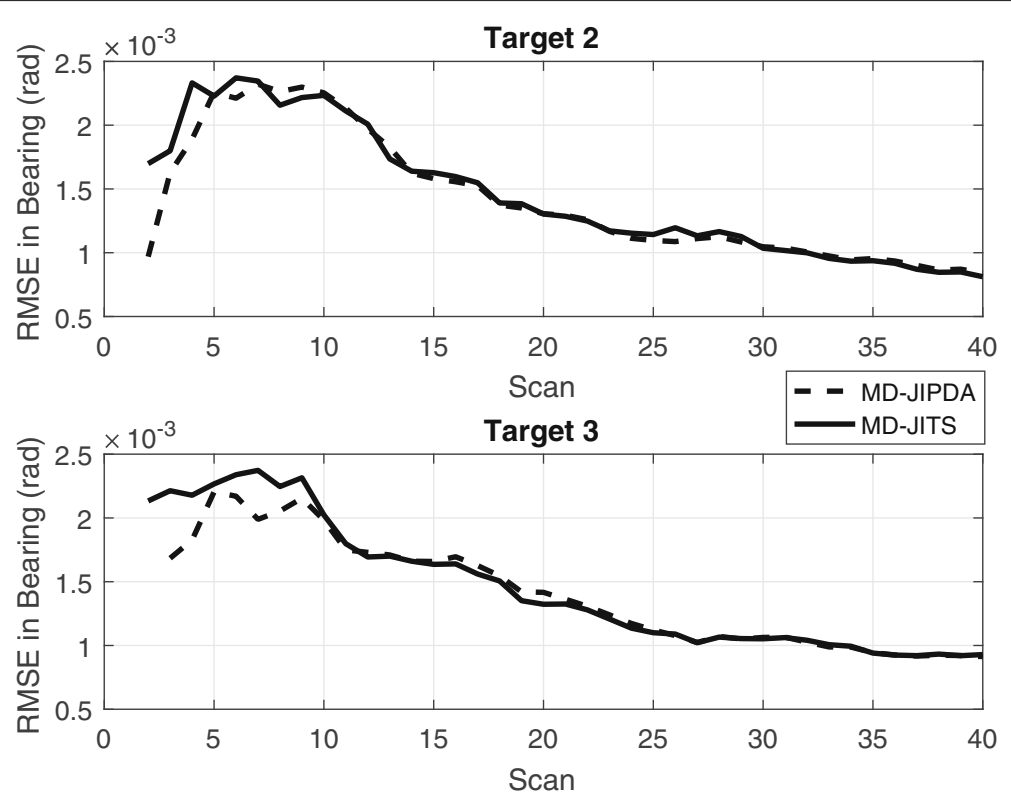

Fig. 5 RMSE in bearing for target 2 and target 3

\section{Conclusions}

In this paper, in order to jointly resolve the measurement origin uncertainty and measurement path model uncertainty in the OTHR system, we developed the MDJITS filter. The main benefit of this approach is that it generates all possible target-oriented measurement combinations with validated measurements considering possible measurement propagation models. The target state information contained in measurements are more efficiently extracted due to one-to-many, track-tomeasurement cell association for this multiple detection problem instead of the existing one-to-one association for single detection. Compared to the widely used PDA framework, MD-JITS is a multi-scan tracker that

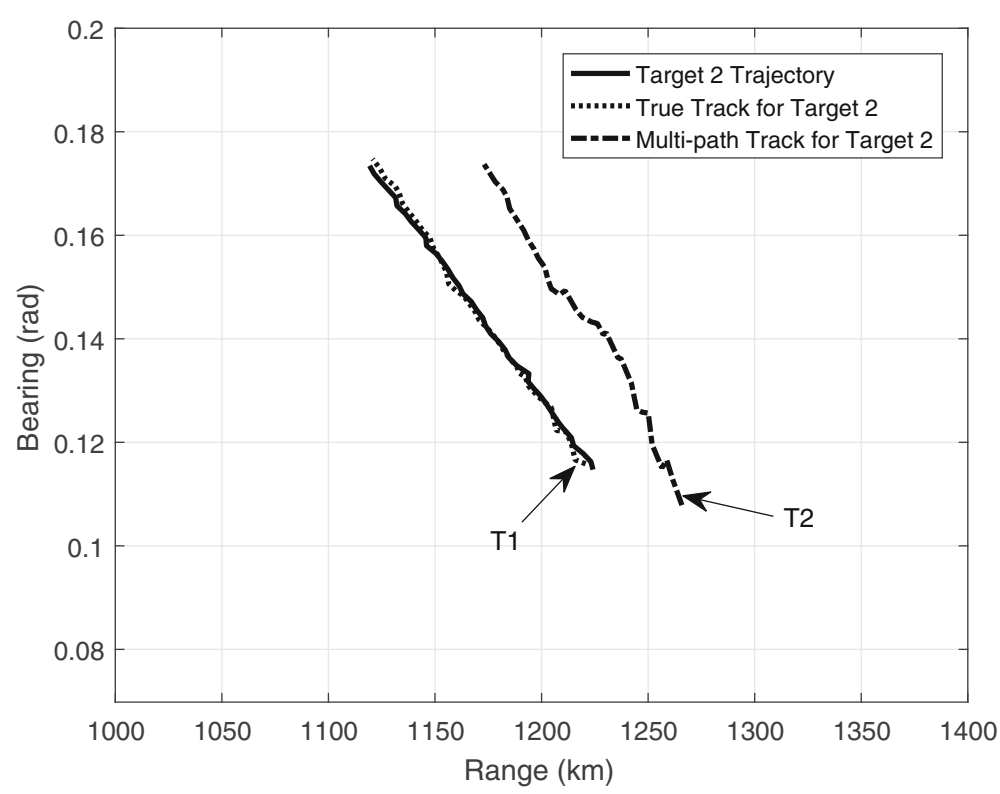

Fig. 6 True and multi-path tracks 
Table 7 Active model statistic

\begin{tabular}{ll}
\hline Track & Active path statistic $[E E, E F, F E, F F]$ \\
\hline T1 & {$[16,16,17,15]$} \\
T2 & {$[19,13,2,0]$} \\
\hline
\end{tabular}

maintains information on the measurement cell history and propagates based on the components, making it more robust in harsh tracking environments.

To demonstrate the superiority of the proposed MDJITS filter, we compared it with the MD-JIPDA filter in a MTT environment using the OTHR system. The simulation results indicate that the new filter outperforms the MD-JIPDA filter for true track confirmation and maintenance and shows the same state estimation accuracy as the MD-JIPDA filter.

There are several aspects worthy for the further work: the method which considers the time varying heights of the ionospheric layers can be inserted into the MD-JITS structure; the more computationally efficient structure of the MD-JITS algorithm is expected to be developed.

\section{Acknowledgements}

The authors would like to thank the anonymous reviewers for the improvement of this paper.

\section{Funding}

This work was supported by Hanwha Systems, Radar R\&D Center through Grant U-17-017.

\section{Availability of data and materials}

The datasets supporting the conclusions of this article are included within the article (and its additional file(s)).

\section{Authors' contributions}

YH made the main contributions to conception and tracking algorithms' design, as well as drafting the article. TLS offered critical suggestions on the algorithms' design, provided significant revising for important intellectual content, and gave final approval of the current version to be submitted. JHL helped to answer the reviewers' questions and revise the manuscript. All the authors read and approved the final manuscript.

\section{Authors' information}

YH was born in Hubei, China, in 1990. He received the B.Sc. degree in mechanical engineering and automation from Wuhan University of Science and Technology, Wuhan, China, in 2013. He is currently working toward a Ph.D. degree in Hanyang University. His research interests include target state estimation and information fusion.

TLS was born in Andong, Korea, in 1952. He received the B.Sc. degree in nuclear engineering from Seoul National University, Seoul, Korea, in 1974, and both the M.Sc. and Ph.D. degrees in aerospace engineering from the University of Texas at Austin, in 1981 and 1983, respectively. From 1974 to 1994, he was with the Agency for Defense Development where his major research topics included missile guidance and control and control systems design, target tracking, and navigation systems analysis. He has been a Professor at the Electronic Systems Engineering Department, Hanyang University, since 1995. His research interests include target state estimation, guidance, navigation, and control. JHL was born in Seoul, Korea, in 1980. He received the B.Sc. degree in computer engineering and an M.Sc. degree in control engineering from Hanyang University in 2006 and 2008, respectively. He is currently working as a senior engineer at Radar R\&D Center of Hanwha Systems.

\section{Competing interests}

The authors declare that they have no competing interests.

\section{Publisher's Note}

Springer Nature remains neutral with regard to jurisdictional claims in published maps and institutional affiliations.

\section{Author details}

${ }^{1}$ Department of Electronic Systems Engineering, Hanyang University, Hanyangdaehak-ro, Ansan, Republic of Korea. ${ }^{2}$ Radar R\&D Center, Hanwha Systems, GyeonggiDong-ro, Yongin, Republic of Korea.

Received: 2 January 2018 Accepted: 4 September 2018

Published online: 20 September 2018

\section{References}

1. Y. Bar-Shalom, X. R. Li, T. Kirubarajan, Estimation with application to tracking and navigation. (Wiley Press, New York, 2001)

2. S. Challa, R. Evans, M. Morelande, D. Musicki, Fundamentals of object tracking. (Cambridge University, United Kingdom, 2011)

3. Y. Bar-Shalom, P. Willett, X. Tian, Tracking and data fusion: a handbook of algorithms. (YBS, Bloomfield, 2011)

4. D. Musicki, R. Evans, S. Stankovic, Integrated probabilistic data association (IPDA). IEEE Trans. Automat. Contr. 39(6), 1237-1241 (1994)

5. D. B. Reid, H. Thomas, An algorithm for tracking multiple targets. IEEE Trans. Automat. Contr. 24(6), 843-854 (1979)

6. Y. Bar-Shalom, E. Tse, Tracking in a cluttered environment with probabilistic. Automatica. 11(5), 451-460 (1975)

7. R. Mahler, Aerosp, Multitarget Bayes filtering via first-order multitarget moments. IEEE Trans. Electron. Syst. 39(4), 1152-1178 (2003)

8. S. Reuter, B. T. Vo, B. N. Vo, The labeled multi-Bernoulli filter. IEEE Trans. Signal Process. 62(12), 3245-3260 (2014)

9. A. M. Aziz, A joint possibilistic data association technique for tracking multiple targets in a cluttered environment. Inform. Sciences. 280(1), 239-260 (2014)

10. H. Zhu, H. Leung, K. V. Yuen, A joint data association, registration, and fusion approach for distributed tracking. Inform. Sciences. 324(10), 186-196 (2015)

11. D. Musicki, R. Evans, Multiscan multitarget tracking in clutter with integrated track splitting filter. IEEE Trans. Aerosp. Electron. Syst. 45(4), 1432-1447 (2009)

12. T. Kurien, Multitarget multisensor tracking: advanced applications. (Artech House, Norwood, 1990)

13. D. Avitzour, A maximum likelihood approach to data association. IEEE Trans. Aerosp. Electron. Syst. 28(2), 560-566 (1996)

14. D. Ciuonzo, S. Horn, A hash-tree based approach for a totally distributed track oriented multi hypothesis tracker. Proc. Int. Conf. IEEE Aerosp, 1-9 (2012)

15. D. Musicki, R. Evans. IEEE Trans. Aerosp. Electron. Syst. 40(3), 1093-1099 (2004)

16. D. Ciuonzo, P. K. Willett, Y. Bar-Shalom, Tracking the tracker from its passive sonar ML-PDA estimates. IEEE Trans. Aerosp. Electron. Syst. 50(1), 573-590 (2014)

17. Y. F. Guo, R. Tharmarasa, S. Rajan, T. L. Song, T. Kirubarajan, Passive tracking in heavy clutter with sensor location uncertainty. IEEE Trans. Aerosp. Electron. Syst. 52(4), 1536-1554 (2016)

18. S. Blackman, Multiple-target tracking with radar applications. (Artech House, Norwood, 1986)

19. D. J. Salmond, Mixture reduction algorithms for target tracking in clutter Proc. Int. Conf. Signal Data Process Small Targets. 1305, 434-445 (1990)

20. D. Musicki, B. L. Scala, R. Evans, Integrated track splitting filter efficient multi-scan single target tracking in clutter. IEEE Trans. Aerosp. Electron. Syst. 43(4), 1409-1425 (2007)

21. T. H. Kim, T. L. Song, Multi-target multi-scan smoothing in clutter. IET Radar Sonar. Navig. 10(7), 1270-1276 (2016)

22. W. G. Pulford, R. Evans, A multipath data association tracker for over-the-horizon radar. IEEE Trans. Aerosp. Electron. Syst. 34(4), 1165-1183 (1998)

23. W. G. Pulford, R. Evans, Authors' reply to 'comments on "multipath data association tracker for over-the-horizon rada"'. IEEE Trans. Aerosp. Electron. Syst. 41(3), 1148-1150 (1998)

24. W. G. Pulford, R. Evans, Authors' reply to 'OTHR multipath tracking with uncertain coordinate registration'. IEEE Trans. Aerosp. Electron. Syst. 40(1), 38-56 (2004) 
25. T. Sathyan, T. J. Chin, S. Arulampalam, D. Suter, A multiple hypothesis tracker for multitarget tracking with multiple simultaneous measurements. IEEE J-STSP. 7(3), 448-460 (2013)

26. J. F. Chen, H. Ma, C. G. Liang, OTHR multipath tracking using the Bernoulli filter. IEEE Trans. Aerosp. Electron. Syst. 50(3), 1974-1990 (2014)

27. X. Tang, X. Chen, M. McDonald, R. Mahler, R. Tharmarasa, T. Kirubarajan, A multiple-detection probability hypothesis density filter. IEEE Trans. Signal Process. 63(8), 2007-2019 (2015)

28. Y. Qin, H. Ma, L. Cheng, L. Yang, X. Q. Zhou, Cardinality balanced multitarget multi-Bernoulli filter for multipath multitarget tracking in over-the-horizon radar. IET Radar Sonar Navig. 10(3), 535-545 (2016)

29. Y. Qin, H. Ma, J. F. Chen, L. Cheng, Gaussian mixture probability hypothesis density filter for multipath multitarget tracking in over-the-horizon radar. EURASIP J. Adv. Signal Process. 2015, 108 (2015)

30. D. Nikolio, Z. Popovic, M. Borenovio, N. Stojkovio, V. Orlic, A. Dzvonkovskaya, B. M. Todorovic, Multi-radar multi-target tracking algorithm for maritime surveillance at OTH distances. Proc. Int. Radar Symp. (IRS)., 1-6 (2016)

31. B. Habtemariam, R. Tharmarasa, T. Thayaparan, M. Mallick, T. Kirubarajan, A multiple-detection joint probabilistic data association filter. IEEE J. Sel. Top. Signal Process. 7(3), 461-471 (2013)

32. Y. Huang, T. L. Song, C. M. Lee, The multiple detection joint integrated track splitting filter. Proc. CIE Int. Conf. Radar, 1-4 (2016)

33. M. Kong, G. H. Wang, J. Bai, Research on target tracking technology of OTHR based on MPDA. Proc. Int. Conf. Radar, 1-4 (2006)

34. H. Geng, Y. Liang, F. Yang, L. F. Xu, Joint estimation of target state and ionosphere state for OTHR based tracking. Proc. Int. Conf. Inf. Fusion, 1270-1277 (2015)

35. H. Geng, Y. Liang, F. Yang, L. F. Xu, Q. Pan, Joint estimation of target state and ionospheric height bias in over-the-horizon radar target tracking. IET Radar Sonar. Navig. 10(7), 1153-1167 (2016)

36. H. Geng, Y. Liang, X. X. Wang, F. Yang, L. F. Xu, Q. Pan, Multi-path multi-rate filter for OTHR based tracking systems. Proc. Int. Conf. Inf. Fusion, 1276-1283 (2016)

37. S. Blackman, R. Popoli, Design and analysis of modern tracking systems. (Artech House, Boston, 1999)

38. F. W. Smith, J. A. Malin, Models for radar scatterer density in terrain images. IEEE Trans. Aerosp. Electron. Syst. AES-22(5), 642-647 (1986)

39. H. Lan, Y. Liang, Q. Pan, An EM algorithm for multipath state estimation in OTHR target tracking. IEEE Trans. Signal Process. 62(11), 2814-2826 (2014)

\section{Submit your manuscript to a SpringerOpen ${ }^{\circ}$ journal and benefit from:}

- Convenient online submission

- Rigorous peer review

- Open access: articles freely available online

- High visibility within the field

- Retaining the copyright to your article

Submit your next manuscript at $\gg$ springeropen.com 